\title{
CIRJE-F-860 \\ Note on an Extension of an Asymptotic Expansion Scheme
}

\author{
Akihiko Takahashi \\ University of Tokyo \\ Masashi Toda \\ Graduate School of Economics, University of Tokyo \\ September 2012; Revised in January and April 2013
}

CIRJE Discussion Papers can be downloaded without charge from:

http://www.cirje.e.u-tokyo.ac.jp/research/03research02dp.html

Discussion Papers are a series of manuscripts in their draft form. They are not intended for circulation or distribution except as indicated by the author. For that reason Discussion Papers may not be reproduced or distributed without the written consent of the author. 


\title{
Note on an Extension of an Asymptotic Expansion Scheme*
}

\author{
Akihiko Takahashi, Masashi Toda \\ Graduate School of Economics, the University of Tokyo \\ 7-3-1, Hongo, Bunkyo-ku, Tokyo, 113-0033, Japan
}

December 18, 2012, This Version: April 9, 2013

\begin{abstract}
This paper presents an extension of a general computational scheme for asymptotic expansions proposed in earlier works by the authors and coworkers. In the earlier works, a new method was developed for the computation of an arbitrary-order expansion with a normal benchmark distribution in a multidimensional diffusion setting. In particular, a new algorithm was proposed for calculating coefficients in an expansion by solving a system of ordinary differential equations. In the present note, by a change of variable technique, and by various ways of setting the perturbation parameters in the expansion, we provide the flexibility of setting the benchmark distribution around which the expansion is made and an automatic way for computation up to any order in the expansion. For instance, we introduce new expansions, called the lognormal expansion and the CEV expansion. We also show some concrete examples with numerical experiments, which imply that a high-order CEV expansion will produce more a precise and stable approximation for option pricing under the SABR model than other approximation methods such as the log-normal expansion and the well-known normal expansion.
\end{abstract}

\section{Introduction}

An asymptotic expansion approach in finance has been developed for the past two decades, which is mathematically justified by Watanabe theory [50] in Malliavin calculus (Malliavin [21], Ikeda and Watanabe [11], Nualart [25]). To the best of our knowledge, the asymptotic expansion technique is firstly applied to finance for evaluation of an average option that is a popular derivative in commodity markets. In [13] and [34], approximation formulas are derived for an average option by an asymptotic method based on lognormal approximations of an average price distribution when the underlying asset price follows a geometric Brownian motion. Yoshida [54] applies a formula derived more generally by his result [53] for the asymptotic expansion of small diffusion processes based on Watanabe theory. Thereafter, asymptotic expansions have been applied to a broad class of problems in finance: See [35], [36], Kunitomo and Takahashi [14], [15], [16], Li [20] Matsuoka, Takahshi and Uchida [23], Osajima [26], Shiraya and Takahashi [28], [29], Shiraya, Takahashi and Toda [30], Shiraya, Takahashi and Yamazaki [31], Shiraya, Takahashi and Yamada [32], Takahashi and Matsushima [37], Takahashi and Saito [38], Takahashi and Yamada [45], [46] Takahashi and Yoshida [47], [48], Takahashi and Uchida [44], and Takahashi and Takehara [39], [40], [41].

For other asymptotic expansion approaches in finance, see also Bayer and Laurence [1], Ben Arous and Laurence [2], Gatheral, Hsu, Laurence, Ouyang, and Wang [9], Fujii and Takahashi [5], [6], [7], Fouque, Papanicolaou and Sircar [8], Henry-Labordere [19], Kato Takahashi and Yamada [12], Kusuoka and Osajima [17], Osajima [27], Siopacha and Teichmann [33], Yamamoto, Sato and Takahashi [51], Yamamoto and Takahashi [52].

In the application of the asymptotic expansion based on Watanabe theory, we need to calculate certain conditional expectations which appear in the expansions and play a key role in computation. In the first

${ }^{*}$ The shorter version will appear in International Journal of Theoretical and Applied Finance. We are very grateful to the anonymous referee for his or her precious comments and suggestions. This research is supported by Center for Advanced Research in Finance $(\mathrm{CARF})$ and the global COE program "The research and training center for new development in mathematics." 
place [53] and [54] have developed the formulas necessary for the second order expansion. Subsequently, [34], [35], [39], [40] and [41] have derived new formulas up to the third order. (Also, multi-dimensional formulas were provided in [34] and [35].) In many applications, these formulas give sufficiently accurate approximation, but in some cases such as in the cases with long maturities or/and with highly volatile underlying variables, the approximation up to the third order may not provide satisfactory accuracies. Thus, the formulas for the higher order computation are desirable.

Recently, [49], [42] and [43] have proposed two alternative computational schemes for any order expansions in an automatic manner. In fact, one of their new methods does not rely on direct evaluation of the conditional expectations, but on solving a certain system of ordinal differential equations with grading structure. Independently, $\mathrm{Li}$ [20] has developed a new computational method for the conditional expectations necessary for high order expansions. As a consequence, their approximations generally showed sufficient accuracy with computation of high order expansions, which was confirmed by numerical experiments.

Furthermore, in terms of approximation it is important to set the limiting or benchmark distribution around which an expansion is made. Typically a normal distribution is chosen, which enables us to compute higher order correction terms due to the nice and well-known Gaussian properties. For (shifted) log-normal local volatility cases in (jump-diffusion) stochastic volatility models, the same technique is applied. (For instance, see [16], [49], [30],[32] [45].)

This paper introduces a change of variable technique in order to obtain the flexibility for setting a benchmark distribution. We also note that a perturbation parameter affects the expansion significantly since different ways of perturbation provide not only different benchmark distributions, but also different correction terms in the given orders. Therefore, we also take various ways of setting a perturbation parameter into account. Through these consideration, we extend a general computational scheme proposed by [49], [42] [43]. Particularly, we construct a scheme that enables us to set a distribution around which we would like to expand a target random variable, and to approximate the target variable up to any order based upon the distribution. As examples, we present new log-normal and $C E V$ expansions for approximations of option prices under a general local-stochastic volatility. In addition, we show those numerical approximations up to the third order for option prices in SABR model, which implies a higher order $C E V$ expansion will provide more precise and robust approximation than other approximation scheme including log-normal and well-known normal expansions.

The next section constructs our new expansion scheme under a general multi-dimensional diffusion setting. Section 3 gives concrete examples with numerical examination. Section 4 concludes. The appendix provides the supplementary numerical results.

\section{Asymptotic Expansions}

\subsection{An Asymptotic Expansion in a General Diffusion Setting}

Let $(W, P)$ be the $r$-dimensional Wiener space. We consider a $d$-dimensional diffusion process $X_{t}=$ $\left(X_{t}^{1}, \cdots, X_{t}^{d}\right)$ which is the solution to the following stochastic differential equation:

$$
\begin{aligned}
& d X_{t}^{j}=V_{0}^{j}\left(X_{t}\right) d t+V^{j}\left(X_{t}\right) d W_{t} \quad(j=1, \cdots, d) \\
& X_{0}=x_{0} \in \mathbf{R}^{d} .
\end{aligned}
$$

Here, $W=\left(W^{1}, \cdots, W^{r}\right)^{\prime}$ is an $r$-dimensional Wiener process where $x^{\prime}$ denotes the transpose of $x$. Also, $V_{0}^{j}: \mathbf{R}^{d} \mapsto \mathbf{R}$ and $V^{j}=\left(V^{j, 1}, \cdots, V^{j, r}\right): \mathbf{R}^{d} \mapsto \mathbf{R}^{r}$ are smooth functions with bounded derivatives of all orders.

Next, let $C: \mathbf{R}^{d} \mapsto \mathbf{R}^{d}$ be a $\mathbf{C}^{2}$-function which has the unique inverse function, $C^{-1}$, and define $\tilde{X}_{t}$ as $\tilde{X}_{t}=C\left(X_{t}\right)$. Then, the dynamics of $\tilde{X}$ is given by

$$
\begin{aligned}
& d \tilde{X}_{t}^{j}=\tilde{V}_{0}^{j}\left(\tilde{X}_{t}\right) d t+\tilde{V}^{j}\left(\tilde{X}_{t}\right) d W_{t} \quad(j=1, \cdots, d), \\
& \tilde{X}_{0}=\tilde{x}_{0},
\end{aligned}
$$


where

$$
\begin{aligned}
& \tilde{V}_{0}^{j}(\tilde{x}):=\sum_{j^{\prime}=1}^{d} \partial_{j^{\prime}} C^{j}\left(C^{-1}(\tilde{x})\right) V_{0}^{j^{\prime}}\left(C^{-1}(\tilde{x})\right)+\frac{1}{2} \sum_{j^{\prime}, k^{\prime}=1}^{d} \partial_{j^{\prime} k^{\prime}} C^{j}\left(C^{-1}(\tilde{x})\right) V^{j^{\prime}}\left(C^{-1}(\tilde{x})\right) V^{k^{\prime}}\left(C^{-1}(\tilde{x})\right)^{\prime}, \\
& \tilde{V}^{j}(\tilde{x}):=\sum_{j^{\prime}=1}^{d} \partial_{j^{\prime}} C^{j}\left(C^{-1}(\tilde{x})\right) V^{j^{\prime}}\left(C^{-1}(\tilde{x})\right)
\end{aligned}
$$

and $\tilde{x}_{0}=C\left(x_{0}\right)$. Here, $C^{j}: \mathbf{R}^{d} \mapsto \mathbf{R}$ denotes the j-th element of $C=\left(C^{1}, \cdots, C^{d}\right) ; \partial_{j^{\prime}} C^{j}(x)$ stands for the first order partial derivative of $C^{j}(x)$ with respect to the $j^{\prime}$-th element of $x$, and $\partial_{j^{\prime} k^{\prime}} C^{j}(x)$ the second order partial derivative of $C^{j}(x)$ with respect to the $j^{\prime}$-th and $k^{\prime}$-th elements of $x ; V^{k^{\prime}}(x)^{\prime}$ is the transpose of $V^{k^{\prime}}(x)$.

Next, we introduce a perturbation parameter $\epsilon \in(0,1]$ as follows:

$$
\begin{aligned}
& \tilde{X}_{t} \mapsto \tilde{X}_{t}^{(\epsilon)} \\
& \tilde{V}_{0}^{j}(\tilde{x}) \mapsto \tilde{V}_{0}^{(\epsilon), j}(\tilde{x}, \epsilon) \\
& \tilde{V}^{j}(\tilde{x}) \mapsto \epsilon \tilde{V}^{j}(\tilde{x}),
\end{aligned}
$$

and hence, the dynamics of $\tilde{X}^{(\epsilon)}$ is expressed as

$$
d \tilde{X}_{t}^{(\epsilon), j}=\tilde{V}_{0}^{(\epsilon), j}\left(\tilde{X}_{t}^{(\epsilon)}, \epsilon\right) d t+\epsilon \tilde{V}^{j}\left(\tilde{X}_{t}^{(\epsilon)}\right) d W_{t} \quad(j=1, \cdots, d) .
$$

Hereafter in this subsection, let us apply the technique developed in [43] to the transformed SDE (3). Firstly, take a smooth function $g: \mathbf{R}^{d} \mapsto \mathbf{R}$ with all of the derivatives having polynomial growth orders. Then, a smooth Wiener functional $g\left(X_{T}^{(\epsilon)}\right)$ has its asymptotic expansion:

$$
g\left(\tilde{X}_{T}^{(\epsilon)}\right) \sim g_{0 T}+\epsilon g_{1 T}+\epsilon^{2} g_{2 T}+\cdots
$$

in $L^{p}$ for every $p>1$ (or in $\mathbf{D}^{\infty}$ ) as $\epsilon \downarrow 0$.

Let $A_{k t}=\left.\frac{1}{k !} \frac{\partial^{k} \tilde{X}_{l}^{(\epsilon)}}{\partial \epsilon^{k}}\right|_{\epsilon=0}$ and $A_{k t}^{j}, j=1, \cdots, d$ denote the $j$-th elements of $A_{k t}$. In particular, $A_{1 t}$ is represented by

$$
A_{1 t}=\int_{0}^{t} Y_{t} Y_{u}^{-1}\left(\partial_{\epsilon} \tilde{V}_{0}\left(\tilde{X}_{u}^{(0)}, 0\right) d u,+\tilde{V}\left(\tilde{X}_{u}^{(0)}\right) d W_{u}\right)
$$

where $\tilde{V}_{0}=\left(\tilde{V}_{0}^{1}, \cdots, \tilde{V}_{0}^{d}\right): \mathbf{R}^{d} \times(0,1] \mapsto \mathbf{R}^{d}$, and $\tilde{V}=\left(\tilde{V}^{1}, \cdots, \tilde{V}^{d}\right): \mathbf{R}^{d} \mapsto \mathbf{R}^{d} \otimes \mathbf{R}^{r} ; \partial_{\epsilon} \tilde{V}_{0}\left(\tilde{X}_{u}^{(0)}, 0\right)$ stands for the partial derivative of $\tilde{V}_{0}\left(\tilde{X}_{u}^{(0)}, \epsilon\right)$ with respect to $\epsilon$ evaluated at $\epsilon=0 ; Y$ denotes the solution to the differential equation:

$$
d Y_{t}=\partial \tilde{V}_{0}\left(\tilde{X}_{t}^{(0)}, 0\right) Y_{t} d t ; Y_{0}=I_{d} .
$$

Here, $\partial \tilde{V}_{0}$ denotes the $d \times d$ matrix whose $(j, k)$-element is $\partial_{k} \tilde{V}_{0}^{j}=\frac{\partial \tilde{V}_{0}^{j}(x, \epsilon)}{\partial x_{k}}, \tilde{V}_{0}^{j}$ is the $j$-th element of $\tilde{V}_{0}$, and $I_{d}$ denotes the $d \times d$ identity matrix. Note that $A_{1 t}$ follows a normal distribution.

For $k \geq 2, A_{k t}^{j}, j=1, \cdots, d$ is recursively determined by the following: ${ }^{1}$

$$
\begin{aligned}
A_{k t}^{j}= & \frac{1}{k !} \int_{0}^{t} \partial_{\epsilon}^{k} \tilde{V}_{0}^{j}\left(\tilde{X}_{u}^{(0)}, 0\right) d u \\
& +\sum_{l=1}^{k} \sum_{\vec{l}_{\beta}, \vec{d}_{\beta}}^{(l)} \frac{1}{(k-l) !} \frac{1}{\beta !} \int_{0}^{t}\left(\prod_{j=1}^{\beta} A_{l_{j} u}^{d_{j}}\right) \partial_{\vec{d}_{\beta}}^{\beta} \partial_{\epsilon}^{k-l} \tilde{V}_{0}^{j}\left(\tilde{X}_{u}^{(0)}, 0\right) d u \\
& +\sum_{\vec{l}_{\beta}, \vec{d}_{\beta}}^{(k-1)} \frac{1}{\beta !} \int_{0}^{t}\left(\prod_{j=1}^{\beta} A_{l_{j} u}^{d_{j}}\right) \partial_{\vec{d}_{\beta}}^{\beta} \tilde{V}^{j}\left(\tilde{X}_{u}^{(0)}\right) d W_{u},
\end{aligned}
$$

\footnotetext{
${ }^{1}$ They can be expressed as the finite sum of iterated multiple Wiener -Itô integrals. See Section 3 of [42] for the detail.
} 
where $\partial_{\epsilon}^{l}=\frac{\partial^{l}}{\partial \epsilon^{l}}, \partial_{\vec{d}_{\beta}}^{\beta}=\frac{\partial^{\beta}}{\partial \tilde{X}_{d_{1}} \cdots \partial \tilde{X}_{d_{\beta}}}$,

$$
\sum_{\vec{l}_{\beta}, \vec{d}_{\beta}}^{(l)}:=\sum_{\beta=1}^{l} \sum_{\vec{l}_{\beta} \in L_{l, \beta}} \sum_{\vec{d}_{\beta} \in\{1, \cdots, d\}^{\beta}},
$$

and

$$
L_{l, \beta}:=\left\{\vec{l}_{\beta}=\left(l_{1}, \cdots, l_{\beta}\right) ; \sum_{j=1}^{\beta} l_{j}=l,\left(l, l_{j}, \beta \in \mathbb{N}\right)\right\} .
$$

Then, $g_{0 T}$ and $g_{1 T}$ can be written as

$$
\begin{aligned}
& g_{0 T}=g\left(\tilde{X}_{T}^{(0)}\right), \\
& g_{1 T}=\sum_{j=1}^{d} \partial_{j} g\left(\tilde{X}_{T}^{(0)}\right) A_{1 T}^{j} .
\end{aligned}
$$

For $n \geq 2, g_{n T}=\left.\frac{1}{n !} \frac{\partial^{n} g\left(\tilde{X}_{T}^{(\epsilon)}\right)}{\partial \epsilon^{n}}\right|_{\epsilon=0}$ is expressed as follows:

$$
g_{n T}=\sum_{\vec{l}_{\beta}, \vec{d}_{\beta}}^{(n)} \frac{1}{\beta !} \partial_{\vec{d}_{\beta}}^{\beta} g\left(\tilde{X}_{T}^{(0)}\right) A_{l_{1} T}^{d_{1}} \cdots A_{l_{\beta} T}^{d_{\beta}} .
$$

Here, we note that each $A_{l t}^{i}(i=1, \cdots, d, l=1,2, \cdots, k, 0 \leq t \leq T)$ has all finite moments due to a grading structure as follows: Consider the stochastic differential equation of the form

$$
d S_{t}=\mu\left(S_{t}, t\right) d t+\sigma\left(S_{t}, t\right) d W_{t} ; S_{0}=s_{0} \in \mathbf{R}^{d}
$$

where $\mu: \mathbf{R}^{d} \times \mathbf{R}^{+} \rightarrow \mathbf{R}^{d}$ and $\sigma: \mathbf{R}^{d} \times \mathbf{R}^{+} \rightarrow \mathbf{R}^{d} \otimes \mathbf{R}^{r}$.

Definition 1. A grading of $\mathbf{R}^{d}$ is a decomposition $\mathbf{R}^{d}=\mathbf{R}^{d_{1}} \times \cdots \times \mathbf{R}^{d_{q}}$ with $d=d_{1}+\cdots+d_{q}$. The coordinates of a point in $\mathbf{R}^{d}$ are always arranged in an increasing order along the subspace $\mathbf{R}^{d_{i}}$, and we set $M_{0}=0$ and $M_{l}=d_{1}+\cdots+d_{l}$ for $1 \leq l \leq q$. We say that the coefficients $\mu$ and $\sigma$ are graded according to the grading $\mathbf{R}^{d}=\mathbf{R}^{d_{1}} \times \cdots \times \mathbf{R}^{d_{q}}$ if $\mu^{i}(s, t)$ and $\sigma_{j}^{i}(s, t), j=1, \cdots, r$ depend upon only through the coordinates $\left(s^{k}\right)_{1 \leq k \leq M_{p}}$ when $M_{p-1} \leq i \leq M_{p}$.

Theorem 1. We assume the coefficients $\mu$ and $\sigma$ in (10) have a Lipschitz lower triangular structure, and are graded according to $\mathbf{R}^{d}=\mathbf{R}^{d_{1}} \times \cdots \times \mathbf{R}^{d_{q}}$. Moreover for $F(s, t)=\mu(s, t)$ or $\sigma_{j}(s, t), j=1, \cdots, r$, we assume $F$ is differentiable in $s$ in $\mathbf{R}^{d}$ and

1. $\left|F^{i}(0, t)\right| \leq Z_{t}$ for $i=1, \cdots, d$

2. $\left|\frac{\partial}{\partial s^{j}} F^{i}(s, t)\right| \leq \hat{Z}_{t}\left(1+|s|^{\theta}\right)$ for all $i, j$

3. $\left|\frac{\partial}{\partial s^{j}} F^{i}(s, t)\right| \leq \zeta$ if $M_{p-1} \leq i, j \leq M_{p}$ for some $p \leq q$

where $\zeta, \theta \geq 0$ are constants, and $Z, \hat{Z}$ are predictable processes such that $\|Z\|_{p}$ and $\|\hat{Z}\|_{p}$ are finite for all $p \geq 1$ where $\|Z\|_{p}=\left\{\int_{0}^{T} E\left[\left|Z_{t}\right|^{p}\right] d t\right\}^{1 / p}$. Then (10) have a unique solution $S$, and for every $p \geq 1$ there are constants $c_{p}$ and $\gamma_{p}$ depending only upon $\left(\zeta, \theta,\left\{\|\hat{Z}\|_{p^{\prime}}\right\}_{p^{\prime} \geq 1}\right)$, such that

$$
\left\|\sup _{0 \leq t \leq T} S_{t}\right\|_{L^{p}} \leq c_{p}\left(s_{0}+\|Z\|_{\gamma_{p}}\right) .
$$

For the detail of the definition and theorem above, see pp.45-47 in Bichteler, Gravereaux and Jacod [3]. Applying Theorem 1 to the system of stochastic differential equations consists of $A_{l t}^{i}(i=1, \cdots, d, l=$ $1, \cdots, k, 0 \leq t \leq T)$ and any products of them, we obtain the following lemma. 
Lemma 1. Each coefficient of the expansion $A_{l t}^{i}(i=1, \cdots, N, l=1, \cdots, k, 0 \leq t \leq T)$ has all finite moments.

(proof) Consider the system of stochastic differential equations which $A_{1}^{1}, \cdots, A_{1}^{d}, A_{1}^{1} A_{1}^{1}, \cdots, A_{1}^{d} A_{1}^{d}$, $A_{2}^{1}, \cdots, A_{2}^{d}, \cdots$ follow. Note that the system of equations is linear and the coefficients of the linear equations are represented by the derivatives at $\epsilon=0$ of $\tilde{V}_{0}\left(X_{u}^{(\epsilon)}, \epsilon\right)$ and $\tilde{V}\left(X_{u}^{(\epsilon)}\right)$ which are bounded in $[0, T]$. Then it is easily shown that the coefficients of the equation have a grading structure and satisfy the conditions in Theorem 1. Hence the coefficients $A_{k t}^{i}$ have all finite moments. $\square$

Next, normalize $g\left(\tilde{X}_{T}^{(\epsilon)}\right)$ to

$$
G^{(\epsilon)}=\frac{g\left(\tilde{X}_{T}^{(\epsilon)}\right)-g_{0 T}}{\epsilon}
$$

for $\epsilon \in(0,1]$. Then,

$$
G^{(\epsilon)} \sim g_{1 T}+\epsilon g_{2 T}+\cdots
$$

in $L^{p}$ for every $p>1$.

Moreover, let

$$
\hat{V}(x, t)=(\partial g(x))^{\prime}\left[Y_{T} Y_{t}^{-1} \tilde{V}(x)\right]
$$

and make the following assumption:

$$
\text { (Assumption 1) } \quad \Sigma_{T}=\int_{0}^{T} \hat{V}\left(\tilde{X}_{t}^{(0)}, t\right) \hat{V}\left(\tilde{X}_{t}^{(0)}, t\right)^{\prime} d t>0 .
$$

Note that $g_{1 T}$ follows a normal distribution with variance $\Sigma_{T}$. The density function of $g_{1 T}$ denoted by $f_{g_{1 T}}(x)$ is expressed as

$$
f_{g_{1 T}}(x)=\frac{1}{\sqrt{2 \pi \Sigma_{T}}} \exp \left(-\frac{(x-c)^{2}}{2 \Sigma_{T}}\right)
$$

where

$$
c=\left(\partial g\left(\tilde{X}_{T}^{(0)}\right)\right)^{\prime} \int_{0}^{T} Y_{T} Y_{t}^{-1} \partial_{\epsilon} \tilde{V}_{0}\left(\tilde{X}_{t}^{(0)}, 0\right) d t .
$$

Hence, (Assumption 1) means that the distribution of $g_{1 T}$ does not degenerate.

Let $\mathcal{S}$ be the real Schwartz space of rapidly decreasing $C^{\infty}$-functions on $\mathbf{R}$ and $\mathcal{S}^{\prime}$ be its dual space.

Next, take $\Phi \in \mathcal{S}^{\prime}$. Then, the asymptotic expansion of a generalized Wiener functional $\Phi\left(G^{(\epsilon)}\right)$ as $\epsilon \downarrow 0$ can be verified by Watanabe theory. (See Watanabe [50], Yoshida [53] or Malliavin [21] for instance.)

In particular, if we take the delta function at $x \in \mathbf{R}, \delta_{x}$ as $\Phi$, we obtain an asymptotic expansion of the density for $G^{(\epsilon)}$. That is, the expectation of $\Phi\left(G^{(\epsilon)}\right)$ is expanded as follows:

$$
\begin{aligned}
\mathbf{E}\left[\Phi\left(G^{(\epsilon)}\right)\right] & =\sum_{n=0}^{N} \epsilon^{n} \sum_{\vec{k}_{\delta}}^{(n)} \frac{1}{\delta !} \mathbf{E}\left[\Phi^{(\delta)}\left(g_{1 T}\right) \prod_{j=1}^{\delta} g_{\left(k_{j}+1\right) T}\right]+o\left(\epsilon^{N}\right) \\
& =\sum_{n=0}^{N} \epsilon^{n} \sum_{\vec{k}_{\delta}}^{(n)} \frac{1}{\delta !} \int_{\mathbf{R}} \Phi^{(\delta)}(x) \mathbf{E}\left[\tilde{X}^{\vec{k}_{\delta}} \mid g_{1 T}=x\right] f_{g_{1 T}}(x) d x+o\left(\epsilon^{N}\right) \\
& =\sum_{n=0}^{N} \epsilon^{n} \sum_{\vec{k}_{\delta}}^{(n)} \frac{1}{\delta !} \int_{\mathbf{R}} \Phi(x)(-1)^{\delta} \frac{d^{\delta}}{d x^{\delta}}\left\{\mathbf{E}\left[\tilde{X}^{\vec{k}_{\delta}} \mid g_{1 T}=x\right] f_{g_{1 T}}(x)\right\} d x+o\left(\epsilon^{N}\right)
\end{aligned}
$$

where $\Phi^{(\delta)}\left(g_{1 T}\right)=\left.\frac{d^{\delta} \Phi(x)}{d x^{\delta}}\right|_{x=g_{1 T}}, \sum_{\vec{k}_{\delta}}^{(n)}=\sum_{\delta=1}^{n} \sum_{\vec{k}_{\delta} \in L_{n, \delta}}$, and

$$
\tilde{X}^{\vec{k}_{\delta}}:=\prod_{j=1}^{\delta} g_{\left(k_{j}+1\right) T}
$$


To compute the asymptotic expansion (12), we need to evaluate the conditional expectations of the form

$$
E\left[\tilde{X}^{\vec{k}_{\delta}} \mid g_{1 T}=x\right]
$$

where $\tilde{X}^{\vec{k}_{\delta}}$ is represented by a product of multiple Wiener-Itô integrals. Previous works such as [34] and [35] provided the conditional expectation formulas necessary for the expansions up to the third order. [42] showed a general scheme for deriving formulas for the higher order expansions. On the other hand, [49] and [43] have introduced an alternative but equivalent computational algorithm for an asymptotic expansion: we compute the unconditional expectations instead of the conditional ones by deriving a system of ordinary differential equations which the expectations satisfy. Thus, we are able to derive high order approximation formulas in an automatic manner.

The next theorem shows a general result for an asymptotic expansion of the density function for $G^{(\epsilon)}$. In particular, the coefficients in the expansion are obtained through the solution of a system of ordinary differential equations(ODEs). The key point is that each ordinary differential equation(ODE) does not involve any higher order terms, and only lower or the same order terms appear in the right hand side of the ODE. Hence, one can easily solve the system of ODEs analytically or numerically.

Theorem 2. The asymptotic expansion of the density function of $G^{(\epsilon)}=\epsilon^{-1}\left[g\left(\tilde{X}_{T}^{(\epsilon)}\right)-g\left(\tilde{X}_{T}^{(0)}\right)\right]$ up to $\epsilon^{N}$-order is given by

$$
\begin{aligned}
f_{G^{(\epsilon)}}(x)= & f_{g_{1 T}}(x) \\
& +\sum_{n=1}^{N} \epsilon^{n}\left(\sum_{m=0}^{3 n} C_{n m} H_{m}\left(x-c, \Sigma_{T}\right)\right) f_{g_{1 T}}(x)+o\left(\epsilon^{N}\right),
\end{aligned}
$$

where $H_{n}(x ; \Sigma)$ is the Hermite polynomial of degree $n$ which is defined as

$$
H_{n}(x ; \Sigma)=(-\Sigma)^{n} e^{x^{2} / 2 \Sigma} \frac{d^{n}}{d x^{n}} e^{-x^{2} / 2 \Sigma},
$$

and

$$
\begin{aligned}
C_{n m}= & \frac{1}{\Sigma_{T}^{m}} \sum_{\vec{k}_{\delta}}^{(m)} \sum_{\vec{l}_{\beta_{1}}^{1}, \vec{d}_{\beta_{1}}^{1}}^{\left(k_{1}+1\right)} \cdots \sum_{\vec{l}_{\beta_{\delta}}^{\delta}, \vec{d}_{\beta_{\delta}}^{\delta}}^{\left(k_{\delta}+1\right)} \frac{1}{\delta !(m-\delta) !} \\
& \times\left.\left(\prod_{j=1}^{\delta} \frac{1}{\beta_{j} !} \partial_{\vec{d}_{\beta_{j}}^{j}}^{\beta_{j}} g\left(\tilde{X}_{T}^{(0)}\right)\right) \frac{1}{i^{m-\delta}} \frac{\partial^{m-\delta}}{\partial \xi^{m-\delta}} \eta_{\vec{l}_{\beta_{1}} \otimes \cdots \otimes \vec{l}_{\beta_{\delta}}^{\delta}}^{\vec{d}_{\beta_{j}} \otimes \otimes \vec{d}_{\beta^{\delta}}^{\delta}}(T ; \xi)\right|_{\xi=0},(i=\sqrt{-1}) .
\end{aligned}
$$


$\eta_{\vec{l}_{\beta}}^{\vec{d}_{\beta}}(T ; \xi)$ are obtained as a solution to the following system of ODEs:

$$
\begin{aligned}
\frac{d}{d t}\left\{\eta_{\vec{l}_{\beta}}^{\vec{d}_{\beta}}(t ; \xi)\right\}= & \sum_{k=1}^{\beta} \frac{1}{l_{k} !} \eta_{\vec{l}_{\beta / k}}^{\vec{d}_{\beta / k}}(t ; \xi) \partial_{\epsilon}^{l_{k}} \tilde{V}_{0}^{d_{k}}\left(\tilde{X}_{t}^{(0)}, 0\right) \\
& +\sum_{k=1}^{\beta} \sum_{l=1}^{l_{k}} \sum_{\vec{m}_{\gamma}, \vec{d}_{\gamma}}^{(l)} \frac{1}{\left(l_{k}-l\right) !} \frac{1}{\eta !} \eta_{\left(\vec{l}_{\beta / k}\right) \otimes \vec{m}_{\gamma}}^{\left(\vec{d}_{\beta / k}\right) \otimes \vec{d}_{\gamma}}(t ; \xi) \partial_{\vec{d}_{\gamma}}^{\gamma} \partial_{\epsilon}^{l_{k}-l} \tilde{V}_{0}^{d_{k}}\left(\tilde{X}_{t}^{(0)}, 0\right) \\
+ & \sum_{k, m=1}^{\beta} \sum_{k<m}^{\left(l_{k}-1\right)} \sum_{\vec{m}_{\gamma}, \vec{d}_{\gamma}}^{\left(l_{m}-1\right)} \frac{1}{\vec{m}_{\delta}, \vec{d}_{\delta}} \frac{1}{\gamma ! \delta !} \eta_{\left(\vec{l}_{\beta / k}, m\right) \otimes \vec{m}_{\gamma} \otimes \vec{m}_{\delta}}^{\left(\vec{d}_{\beta / k}\right.}(t ; \xi) \\
& \times \partial_{\vec{d}_{\gamma}}^{\gamma} \tilde{V}^{d_{k}}\left(\tilde{X}_{t}^{(0)}\right) \partial_{\vec{d}_{\delta}}^{\delta} \tilde{V}^{d_{m}}\left(\tilde{X}_{t}^{(0)}\right) \\
+ & (i \xi) \sum_{k=1}^{\beta} \sum_{\vec{m}_{\gamma}, \overrightarrow{\vec{d}}_{\gamma}}^{\left(l_{k}-1\right)} \frac{1}{\gamma !} \eta_{\left(\vec{l}_{\beta / k}\right) \otimes \vec{m}_{\gamma}}^{\left(\vec{d}_{\beta}\right) \otimes \vec{d}_{\gamma}}(t ; \xi) \partial_{\vec{d}_{\gamma}}^{\gamma} \tilde{V}^{d_{k}}\left(\tilde{X}_{t}^{(0)}\right) \hat{V}\left(\tilde{X}_{t}^{(0)}, t\right) \\
\eta_{\vec{l}_{\beta}}^{\vec{d}_{\beta}}(0 ; \xi)= & 0 \text { for }\left(\vec{l}_{\beta}, \vec{d}_{\beta}\right) \neq(\emptyset, \emptyset), \eta_{(\emptyset)}^{(\emptyset)}(t ; \xi)=1 .
\end{aligned}
$$

Here, we use the following notations:

$$
\begin{aligned}
\vec{l}_{\beta / k} & :=\left(l_{1}, \cdots, l_{k-1}, l_{k+1}, \cdots, l_{\beta}\right) \\
\vec{l}_{\beta / k, n} & :=\left(l_{1}, \cdots, l_{k-1}, l_{k+1}, \cdots, l_{n-1}, l_{n+1}, \cdots, l_{\beta}\right), 1 \leq k<n \leq \beta \\
\vec{l}_{\beta} \otimes \vec{m}_{\gamma} & :=\left(l_{1}, \cdots, l_{\beta}, m_{1}, \cdots, m_{\gamma}\right)
\end{aligned}
$$

for $\vec{l}_{\beta}=\left(l_{1}, \cdots, l_{\beta}\right)$ and $\vec{m}_{\gamma}=\left(m_{1}, \cdots, m_{\gamma}\right)$.

The proof is given in Sections 3 and 5 of [43].

Remark 1. Due to $\eta_{(\emptyset)}^{(\emptyset)}(t ; \xi)=1$ and the hierarchical structure of the ODEs with respect to $l=\sum_{j=1}^{\beta} l_{j}$, one can easily solve these ODEs successively from lower order terms to higher order terms with initial conditions $\eta_{\vec{l}_{\beta}}^{\vec{d}_{\beta}}(0 ; \xi)=0$ for $\left(\vec{l}_{\beta}, \vec{d}_{\beta}\right) \neq(\emptyset, \emptyset)$. For instance, $\eta_{(1)}^{j}, \eta_{(1,1)}^{j, k}$ and $\eta_{(2)}^{j}$ are evaluated in the following order:

$$
\eta_{(1)}^{j} \rightarrow \eta_{(1,1)}^{j, k} \rightarrow \eta_{(2)}^{j}
$$

Remark 2. We can extend Lemma 1 in [43], which easily leads to the asymptotic expansion of a multidimensional density function in the same manner as for the one dimensional case appearing in the above theorem. That is, we obtain the following result as an extension of Lemma 1 in [43].

Let $(\Omega, \mathcal{F}, P)$ be a probability space. Suppose that $X \in L^{2}(\Omega, P)$ and $\vec{Z}$ is a d-dimensional random variable with Gaussian distribution with mean $\overrightarrow{0}$ and variance-covariance matrix $\underline{\Sigma}$. Then, the conditional expectation $\mathbf{E}[X \mid \vec{Z}=\vec{x}]$ for $\vec{x} \in \mathbf{R}^{d}$ has the following expansion in $L^{2}\left(\mathbf{R}^{d}, \vec{\mu}\right)$ where $\vec{\mu}$ is the Gaussian measure on $\mathbf{R}^{d}$ with mean $\overrightarrow{0}$ and variance $\underline{\Sigma}$ :

$$
\mathbf{E}[X \mid \vec{Z}=\vec{x}]=\sum_{|\vec{n}|=0}^{\infty} a_{\vec{n} !} H_{\vec{n}}(\vec{x}: \underline{\Sigma})
$$

where $\vec{n}=\left(n_{1}, n_{2}, \cdots, n_{d}\right),|\vec{n}|=n_{1}+n_{2}+\cdots+n_{d}, \vec{n} !=n_{1} ! n_{2} ! \cdots n_{d} !$ and

$$
a_{\vec{n}}=\left.\frac{1}{\vec{n}} \frac{1}{i^{|\vec{n}|}} \frac{\partial^{\vec{n}}}{\partial \vec{\xi}}\right|_{\vec{\xi}=\overrightarrow{0}}\left\{e^{\frac{1}{2} \vec{\xi}^{\top} \underline{\Sigma} \vec{\xi}} \mathbf{E}\left[e^{\vec{\xi}^{\top} \vec{Z}} X\right]\right\}
$$

Here, $H_{\vec{n}}(\vec{x}: \underline{\Sigma})$ stands for the d-dimensional multiple Hermite polynomial of degree $|\vec{n}|$ with $\vec{n}=\left(n_{1}, n_{2}, \cdots, n_{d}\right)$ :

$$
H_{\vec{n}}(\vec{x}: \underline{\Sigma})=\frac{1}{n[\vec{x}: \underline{\Sigma}]}\left(-\frac{\partial}{\partial_{x_{1}}}\right)\left(-\frac{\partial}{\partial_{x_{2}}}\right) \cdots\left(-\frac{\partial}{\partial_{x_{d}}}\right) n[\vec{x}: \underline{\Sigma}] ; \vec{x}=\left(x_{1}, x_{2}, \cdots, x_{d}\right)
$$


where

$$
n[\vec{x}: \underline{\Sigma}]=\frac{1}{(2 \pi)^{d / 2}|\underline{\Sigma}|^{1 / 2}} \exp \left\{-\frac{1}{2} \vec{x}^{\top} \underline{\Sigma}^{-1} \vec{x}\right\} .
$$

Indeed, since the system of Hermite polynomials:

$$
\left\{H_{\vec{n}}(\vec{x}: \underline{\Sigma}): \vec{n}=\left(n_{1}, n_{2}, \cdots, n_{d}\right), n_{i}=0,1,2 \cdots(i=1,2, \cdots, d)\right\}
$$

is an orthogonal basis of $L^{2}\left(\mathbf{R}^{d}, \vec{\mu}\right)$, and $E[X \mid \vec{Z}=\vec{x}] \in L^{2}\left(\mathbf{R}^{d}, \vec{\mu}\right)$, we have the following unique expansion of $E[X \mid \vec{Z}=\vec{x}]$ in $L^{2}\left(\mathbf{R}^{d}, \vec{\mu}\right)$ :

$$
\mathbf{E}[X \mid \vec{Z}=\vec{x}]=\sum_{|\vec{n}|=0}^{\infty} a_{\vec{n}} H_{\vec{n}}(\vec{x}: \underline{\Sigma}) .
$$

On the other hand, we know the relation:

$$
\sum_{|\vec{j}|=0}^{\infty} \frac{(i \vec{\xi} \vec{j}}{\vec{j} !} \tilde{H}_{\vec{j}}(\vec{x}: \underline{\Sigma})=e^{i \vec{\xi}^{\top} \vec{x}} e^{\frac{1}{2} \vec{\xi}^{\top} \underline{\Sigma} \vec{\xi}},
$$

and hence,

$$
e^{\vec{\xi}^{\top} \vec{x}}=e^{-\frac{1}{2} \vec{\xi}^{\top} \underline{\Sigma} \vec{\xi}} \sum_{|\vec{j}|=0}^{\infty} \frac{(i \vec{\xi})^{j}}{\vec{j} !} \tilde{H}_{\vec{j}}(\vec{x}: \underline{\Sigma}),
$$

where

$$
\begin{aligned}
\tilde{H}_{\vec{n}}(\vec{x}: \underline{\Sigma}) & =\frac{1}{n[\vec{x}: \underline{\Sigma}]}\left(-\frac{\partial}{\partial_{y_{1}}}\right)\left(-\frac{\partial}{\partial_{y_{2}}}\right) \cdots\left(-\frac{\partial}{\partial_{y_{d}}}\right) n[\vec{x}: \underline{\Sigma}] \\
\vec{y} & =\left(y_{1}, y_{2}, \cdots, y_{d}\right)=\underline{\Sigma}^{-1} \vec{x} .
\end{aligned}
$$

Therefore,

$$
\begin{aligned}
e^{\frac{1}{2} \vec{\xi}^{\top} \underline{\Sigma} \vec{\xi}} \mathbf{E}\left[e^{\vec{\xi}^{\top} \vec{Z}} X\right]= & e^{\frac{1}{2} \vec{\xi}^{\top} \Sigma \vec{\xi}} \mathbf{E}\left[e^{\vec{\xi}^{\top} \vec{Z}} \mathbf{E}[X \mid \vec{Z}=\vec{x}]\right] \\
= & \int_{\mathbf{R}^{d}}\left\{\sum_{|\vec{j}|=0}^{\infty} \tilde{H}_{\vec{n}}(\vec{x}: \underline{\Sigma})(i \vec{\xi})^{\vec{j}}\right\}\left\{\sum_{|\vec{n}|=0}^{\infty} a_{\vec{n}} H_{\vec{n}}(\vec{x}: \underline{\Sigma})\right\} \mu(d \vec{x}) \\
= & \sum_{\substack{|\vec{n}|=0\\
}}^{\infty} \vec{n} ! a_{\vec{n}} i^{|\vec{n}|} \mid \vec{\xi}^{n} ; \\
& \left(\vec{\xi}^{\vec{n}}=\xi_{1}^{n_{1}} \xi_{2}^{n_{2}} \cdots \xi_{d}^{n_{d}}\right),
\end{aligned}
$$

and making $\vec{n}=\left(n_{1}, \cdots, n_{d}\right)$-th order differentiation of both sides in (24) with respect to $\vec{\xi}=\left(\xi_{1}, \cdots, \xi_{d}\right)$ at $\vec{\xi}=\overrightarrow{0}$, we obtain (19) and hence the result, (18) - (21).

\subsection{Applications to Option Pricing}

Given the above theorem for an approximation of the density of $G^{(\epsilon)}$, we can easily derive approximation formulas for option prices under various models.

For instance, let us evaluate a plain-vanilla call option on the underlying asset whose price process is given by $X^{1}$ where $X^{1}$ denotes the first element of $X$. We first determine a change of variable function, $C$ such that

$$
C(x)=\left(C_{1}\left(x^{1}\right), C_{d-1}\left(x^{2}, \cdots, x^{d}\right)\right),
$$


where $x^{j}$ denotes the $j$-th element of $x \in \mathbf{R}^{d}$, and $C_{1}: \mathbf{R} \mapsto \mathbf{R}$ and $C_{d-1}: \mathbf{R}^{d-1} \mapsto \mathbf{R}^{d-1}$ are some invertible functions. Then, we have $\tilde{X}_{t}=C\left(X_{t}\right)$ for all $t \in[0, T]$.

Next, we introduce a perturbation parameter $\epsilon \in[0,1]$ to get $\tilde{X}_{t}^{(\epsilon)}=\left(\tilde{X}_{t}^{(\epsilon), 1}, \cdots, \tilde{X}_{t}^{(\epsilon), d}\right)$ for all $t \in[0, T]$ as in (3), and define $X_{T}^{(\epsilon), 1}=C^{-1}\left(\tilde{X}_{T}^{(\epsilon), 1}\right)$, and in particular, $X^{1}=C^{-1}\left(\tilde{X}_{T}^{(1), 1}\right)$. Also, we set a smooth function $g: \mathbf{R}^{d} \mapsto \mathbf{R}$ as $g(x)=x^{1}$ for $x=\left(x^{1}, \cdots, x^{d}\right)$ where $g$ appears in (4) of the previous subsection.

Let us consider an approximation of the call option price, $\operatorname{Call}^{(\epsilon)}(K, T)$ with maturity $T$ and strike price $K$, whose payoff is given by

$$
\left(X_{T}^{(\epsilon), 1}-K\right)_{+}:=\max \left\{X_{T}^{(\epsilon), 1}-K, 0\right\} .
$$

Then, we obtain an approximation of the call price as follows:

$$
\begin{aligned}
\operatorname{Call}^{(\epsilon)}(K, T) & =P(0, T) \mathbf{E}\left[\left(C_{1}^{-1}\left(\tilde{X}_{T}^{(\epsilon), 1}\right)-K\right)_{+}\right]=P(0, T) \mathbf{E}\left[\left(C_{1}^{-1}\left(\epsilon G^{(\epsilon)}+\tilde{X}_{T}^{(0), 1}\right)-K\right)_{+}\right] \\
& \approx P(0, T) \int_{y^{(\epsilon)}}^{\infty}\left(C_{1}^{-1}\left(\epsilon x+\tilde{X}_{T}^{(0), 1}\right)-K\right) f_{G^{(\epsilon)}, N}(x) d x
\end{aligned}
$$

where

$$
\begin{aligned}
G^{(\epsilon)} & =\frac{\left(\tilde{X}_{T}^{(\epsilon), 1}-\tilde{X}_{T}^{(0), 1}\right)}{\epsilon}, \\
y^{(\epsilon)} & =\frac{C_{1}(K)-\tilde{X}_{T}^{(0), 1}}{\epsilon} .
\end{aligned}
$$

Here, $P(0, T)$ stands for the price at time 0 of a zero coupon bond with maturity $T$, and $f_{G^{(\epsilon), N}}$ denotes the asymptotic expansion of the density for $G^{(\epsilon)}$ up to $\epsilon^{N}$-th order:

$$
f_{G^{(\epsilon), N}}(x)=f_{g_{1 T}}(x)+\sum_{n=1}^{N} \epsilon^{n}\left(\sum_{m=0}^{3 n} C_{n m} H_{m}\left(x-c, \Sigma_{T}\right)\right) f_{g_{1 T}}(x),
$$

which is obtained from the first and second terms of (14) in Theorem 2.

Particularly, when $\epsilon=1$, the payoff is given by

$$
\left(X_{T}^{1}-K\right)_{+}=\left(X_{T}^{(1), 1}-K\right)_{+} .
$$

Then, an approximation of the call price, $\operatorname{Call}(K, T) \equiv \operatorname{Call}^{(1)}(K, T)$ with maturity $T$ and strike price $K$ is given as

$$
\begin{aligned}
\operatorname{Call}(K, T) & =P(0, T) \mathbf{E}\left[\left(C_{1}^{-1}\left(\tilde{X}_{T}^{1}\right)-K\right)_{+}\right]=P(0, T) \mathbf{E}\left[\left(C_{1}^{-1}\left(G^{(1)}+\tilde{X}_{T}^{(0), 1}\right)-K\right)_{+}\right] \\
& \approx P(0, T) \int_{y}^{\infty}\left(C_{1}^{-1}\left(x+\tilde{X}_{T}^{(0), 1}\right)-K\right) f_{G^{(1)}, N}(x) d x
\end{aligned}
$$

where

$$
\begin{aligned}
G^{(1)} & =\tilde{X}_{T}^{(1), 1}-\tilde{X}_{T}^{(0), 1}, \\
y & =C_{1}(K)-\tilde{X}_{T}^{(0), 1},
\end{aligned}
$$

and $f_{G^{(1)}, N}$ is given by

$$
f_{G^{(1), N}}(x)=f_{g_{1 T}}(x)+\sum_{n=1}^{N}\left(\sum_{m=0}^{3 n} C_{n m} H_{m}\left(x-C, \Sigma_{T}\right)\right) f_{g_{1 T}}(x) .
$$

Various approximation formulas could be obtained through choice of the change of variable function $C$ or/and the way to setting the perturbation parameter $\epsilon$ in $\tilde{V}_{0}^{j}\left(\tilde{X}_{t}^{(\epsilon)}, \epsilon\right)$ of (3): for instance, we can set $\tilde{V}_{0}^{j}\left(\tilde{X}_{t}^{(\epsilon)}\right), \epsilon \tilde{V}_{0}^{j}\left(\tilde{X}_{t}^{(\epsilon)}\right), \epsilon^{2} \tilde{V}_{0}^{j}\left(\tilde{X}_{t}^{(\epsilon)}\right), \cdots$. Then, the limiting distribution of the underlying asset price may become normal, log-normal, shifted log-normal, non-central chi-square, and so on. Given this general discussion, the next subsection will illustrate option pricing under a local-stochastic volatility model. 


\subsubsection{Option Pricing under Local-Stochastic Volatility Model}

We assume the underlying process is the unique solution to the following SDE:

$$
\begin{aligned}
& d S_{t}=\sigma\left(X_{t}\right) h\left(S_{t}\right) d W_{t}, \\
& d X_{t}^{j}=V_{0}^{j}\left(X_{t}\right) d t+V^{j}\left(X_{t}\right) d W_{t} \quad(j=2, \cdots, d), \\
& S_{0}=s_{0} \in \mathbf{R}, \quad X_{0}=x_{0} \in \mathbf{R}^{d-1},
\end{aligned}
$$

where $\sigma: \mathbf{R}^{d-1} \rightarrow \mathbf{R}^{r}, h: \mathbf{R} \rightarrow \mathbf{R}$, and $W$ is an $r$-dimensional Wiener process. Then, we evaluate a call option with strike $K$ and maturity $T$, whose underlying price process is given by $S$. For simplicity, we set the zero discount interest rate. Then, the call price $\operatorname{Call}(K, T)$ is expressed as

$$
\operatorname{Call}(K, T)=\mathbf{E}\left[\left(S_{T}-K\right)_{+}\right] .
$$

Firstly, let

$$
C(x)=\left(C_{1}\left(x^{1}\right), x^{2}, \cdots, x^{d}\right), x=\left(x^{1}, x^{2}, \cdots, x^{d}\right),
$$

where $C_{1}: \mathbf{R} \rightarrow \mathbf{R}$ be an invertible $\mathbf{C}^{2}$-function. Then, $\tilde{S}_{t}=C_{1}\left(S_{t}\right)$, and the dynamics of $\tilde{S}$ is given by

$$
d \tilde{S}_{t}=\frac{1}{2}\left\|\sigma\left(X_{t}\right)\right\|^{2} h\left(C_{1}^{-1}\left(\tilde{S}_{t}\right)\right)^{2} C_{1}^{\prime \prime}\left(C_{1}^{-1}\left(\tilde{S}_{t}\right)\right) d t+\sigma\left(X_{t}\right) C_{1}^{\prime}\left(C_{1}^{-1}\left(\tilde{S}_{t}\right)\right) d W_{t}, \quad \tilde{s}_{0}=C_{1}\left(s_{0}\right),
$$

where $C_{1}^{\prime}(x)$ and $C_{1}^{\prime \prime}(x)$ stand for the first and second order derivatives of $C_{1}(x)$ with respect to $x$, respectively.

Next, we introduce a perturbation parameter $\epsilon$ as follows:

$$
\begin{aligned}
& d \tilde{S}_{t}^{(\epsilon)}=\frac{\eta(\epsilon)}{2}\left\|\sigma\left(X_{t}^{(\epsilon)}\right)\right\|^{2} h\left(C^{-1}\left(\tilde{S}_{t}^{(\epsilon)}\right)\right)^{2} C^{\prime \prime}\left(C^{-1}\left(\tilde{S}_{t}^{(\epsilon)}\right)\right) d t+\epsilon \sigma\left(X_{t}^{(\epsilon)}\right) C^{\prime}\left(C^{-1}\left(\tilde{S}_{t}^{(\epsilon)}\right)\right) d W_{t}, \\
& d X_{t}^{(\epsilon), j}=V_{0}^{j}\left(X_{t}^{(\epsilon)}, \epsilon\right) d t+\epsilon V^{j}\left(X_{t}^{(\epsilon)}\right) d W_{t} \quad(j=1, \cdots, d),
\end{aligned}
$$

where $\eta(\epsilon)=\epsilon^{j}$ and $j$ is a nonnegative integer such as $j=0,1,2, \cdots$. Note that

$$
S_{t}=C_{1}^{-1}\left(\tilde{S}_{t}\right)=C_{1}^{-1}\left(\tilde{S}_{t}^{(1)}\right)
$$

According to Theorem 2, we already have an asymptotic expansion of the density function for $G^{(\epsilon)}=$ $\frac{\tilde{S}_{T}^{(\epsilon)}-\tilde{S}_{T}^{(0)}}{\epsilon}$ up to $\epsilon^{N}$-order, which is denoted by $f_{G^{(\epsilon)}, N}(x)$ in $(27)$.

Therefore, an approximation formula of the call price is given as follows:

$$
\begin{aligned}
\operatorname{Call}(K, T) & =\mathbf{E}\left[\left(S_{T}-K\right)_{+}\right]=\mathbf{E}\left[\left(C_{1}^{-1}\left(\tilde{S}_{T}^{(1)}\right)-K\right)_{+}\right] \\
& \approx \int_{y}^{\infty}\left(C_{1}^{-1}\left(x+\tilde{S}_{T}^{(0)}\right)-K\right) f_{G^{(1)}, N}(x) d x,
\end{aligned}
$$

where $y=C_{1}(K)-\tilde{S}_{T}^{(0)}$.

A simple example is the case that the local volatility function is linear:

$$
\begin{aligned}
& d S_{t}=\sigma\left(X_{t}\right) S_{t} d W_{t}, \\
& d X_{t}^{j}=V_{0}^{j}\left(X_{t}\right) d t+V^{j}\left(X_{t}\right) d W_{t} \quad(j=2, \cdots, d) .
\end{aligned}
$$

Let

$$
C(x)=\left(\log x^{1}, x^{2}, \cdots, x^{d}\right), x=\left(x^{1}, x^{2}, \cdots, x^{d}\right),
$$

and set $\eta(\epsilon)=\epsilon^{j}$ where $j$ is 0,1 or 2 . Then, we have $\tilde{S}_{t}^{(\epsilon)}=\log S_{t}^{(\epsilon)}$, whose dynamics is expressed as

$$
\begin{aligned}
& d \tilde{S}_{t}^{(\epsilon)}=-\frac{\epsilon^{j}}{2} \sigma\left(X_{t}^{(\epsilon)}\right)^{2} d t+\epsilon \sigma\left(X_{t}^{(\epsilon)}\right) d W_{t}, \\
& d X_{t}^{(\epsilon), j}=V_{0}^{j}\left(X_{t}^{(\epsilon)}, \epsilon\right) d t+\epsilon V^{j}\left(X_{t}^{(\epsilon)}\right) d W_{t} \quad(j=1, \cdots, d) .
\end{aligned}
$$

This case includes existing researches such as [40], [49], [41], [42], [43], [45]. 


\section{$3 \quad$ Examples}

This section will provide concrete examples with numerical examination.

\subsection{Constant Elasticity of Variance(CEV) Model}

The first example is on the well-known CEV model (Cox [4]) :

$$
d S_{t}=\sigma\left(S_{t}^{\beta} S_{0}^{1-\beta}\right) d W_{t} ; \sigma_{0}>0, S_{0}>0,
$$

where $\beta \in[0,1]$ and $W$ is a one-dimensional Wiener process. Note that the term $S_{0}^{1-\beta}$ makes the level of $\sigma$ is of the same order for different $\beta$. For $x>0$, let us take the change of variable function to be $C(x)=\log \left(x / S_{0}\right)$, that is $x=C^{-1}(\tilde{x})=S_{0} \exp (\tilde{x})$. Hence, $\tilde{S}_{t}=\log \frac{S_{t}}{S_{0}}$ and we have

$$
d \tilde{S}_{t}=-\frac{1}{2} \sigma^{2} e^{2(\beta-1) \tilde{S}_{t}} d t+\sigma e^{(\beta-1) \tilde{S}_{t}} d W_{t} .
$$

Next, we introduce a perturbation $\epsilon \in[0,1]$, again as follows:

$$
d \tilde{S}_{t}^{(\epsilon)}=-\frac{\eta(\epsilon)}{2} \sigma^{2} e^{2(\beta-1) \tilde{S}_{t}^{(\epsilon)}} d t+\epsilon \sigma e^{(\beta-1) \tilde{S}_{t}^{(\epsilon)}} d W_{t},
$$

where $\eta(\epsilon)=\epsilon^{j}$ and $j$ is a nonnegative integer.

Because

$$
S_{T}=C^{-1}\left(\tilde{S}_{T}^{(1)}\right)=S_{0} \exp \left(\tilde{S}_{T}^{(1)}\right)=S_{0} \exp \left(G^{(1)}+S_{T}^{(0)}\right)
$$

an approximation formula of the call price with strike $K$ and maturity $T$ is given as follows:

$$
\begin{aligned}
\operatorname{Call}(K, T) & =\mathbf{E}\left[\left(S_{T}-K\right)_{+}\right]=\mathbf{E}\left[\left(S_{0} \exp \left(G^{(1)}+\tilde{S}_{T}^{(0)}\right)-K\right)_{+}\right] \\
& \approx \int_{y}^{\infty}\left(S_{0} \exp \left(x+\tilde{S}_{T}^{(0)}\right)-K\right) f_{G^{(1)}, N}(x) d x ; \\
y & =C(K)-\tilde{S}_{T}^{(0)}=\log \frac{K}{S_{0}}-\tilde{S}_{T}^{(0)} .
\end{aligned}
$$

Note that $f_{g_{1 T}}$, the first term in the asymptotic expansion of the density $f_{G^{(\epsilon)}}$ is a normal density, and hence the underlying asset price is expanded around a log-normal distribution. Thus, we could call this case a log-normal asymptotic expansion. We also remark that the case of $\eta(\epsilon)=\epsilon^{0}=1$ is harder to be evaluated than the other cases, which is essentially due to difficulty in computation of $\tilde{S}_{t}^{(0)}$ for $\eta(\epsilon)=1$.

\subsection{The $\lambda$-SABR Model}

Let us consider a stochastic volatility model, so-called $\lambda$-SABR Model [19]:

$$
\begin{aligned}
& d S_{t}=\sigma_{t}\left(S_{t}^{\beta} S_{0}^{1-\beta}\right) d W_{t}^{1} ; S_{0}>0, \\
& d \sigma_{t}=\lambda\left(\theta-\sigma_{t}\right) d t+\nu \sigma_{t}\left(\rho d W_{t}^{1}+\sqrt{1-\rho^{2}} d W_{t}^{2}\right) ; \sigma_{0}>0,
\end{aligned}
$$

where $\beta \in[0,1], \lambda \geq 0, \theta>0, \nu>0, \rho \in[-1,1]$ and $W=\left(W^{1}, W^{2}\right)$ is a two dimensional Wiener process.

Remark 3. Previous works such as [49], [42] and [43] have considered an asymptotic expansion based on the following perturbed process, where the change of variable function, $C$ is set by $C(x)=x$ :

$$
\begin{aligned}
& d S_{t}^{(\epsilon)}=\epsilon \sigma_{t}\left(S_{t}^{(\epsilon)}\right)^{\beta} d W_{t}^{1} ; S_{0}^{(\epsilon)}=S_{0}>0, \\
& d \sigma_{t}^{(\epsilon)}=\lambda\left(\theta-\sigma_{t}^{(\epsilon)}\right) d t+\epsilon \nu \sigma_{t}^{(\epsilon)} d W_{t}^{2} ; \sigma_{0}^{(\epsilon)}=\sigma_{0}>0
\end{aligned}
$$


From a viewpoint of mathematical justification of our asymptotic expansion, we may consider a smooth and bounded version of the local volatility function, $x^{\beta}$ in the above model as follows:

$$
\begin{aligned}
d S_{t}^{(\epsilon)} & =\epsilon \sigma_{t} g_{1}\left(S_{t}^{(\epsilon)}\right) d W_{t}^{1} \\
d \sigma_{t}^{(\epsilon)} & =\lambda\left(\theta-\sigma_{t}^{(\epsilon)}\right) d t+\epsilon \nu \sigma_{t}^{(\epsilon)} d W_{t}^{2}
\end{aligned}
$$

where for prefixed very small $K_{3}>0$ and very large $K_{1}>K_{2}\left(>K_{3}\right)$,

$$
\begin{aligned}
g_{1}(x) & =h_{1}(x) g_{2}(x)+h_{2}(x) K_{1}^{\beta}, \\
g_{2}(x) & =h_{3}(x) x^{\beta}, \\
h_{1}(x) & =\frac{\psi\left(K_{1}-x\right)}{\psi\left(x-K_{2}\right)+\psi\left(K_{1}-x\right)}, 0<K_{2}<K_{1}, \\
h_{2}(x) & =\frac{\psi\left(x-K_{2}\right)}{\psi\left(x-K_{2}\right)+\psi\left(K_{1}-x\right)}, 0<K_{2}<K_{1}, \\
h_{3}(x) & =\frac{\psi(x)}{\psi(x)+\psi\left(K_{3}-x\right)}, 0<K_{3}<K_{2}<K_{1}, \\
\psi(x) & =e^{-1 / x} \text { for } x>0, \psi(x)=0 \text { for } x \leq 0 .
\end{aligned}
$$

Note that the local volatility function $g_{1}(x)$ shows the following feature:

$$
\begin{aligned}
g_{1}(x) & =0, \text { if } x \leq 0 \\
& =h_{3}(x) x^{\beta}, \text { if } 0<x \leq K_{3} \\
& =x^{\beta}, \text { if } K_{3}<x \leq K_{2} \\
& =h_{1}(x) x^{\beta}+h_{2}(x) K_{1}^{\beta}, \text { if } K_{2}<x \leq K_{1} \\
& \left.=K_{1}^{\beta}, \text { if } x>K_{1} \text { (constant }\right) .
\end{aligned}
$$

Hence, this model is be regarded as a smooth and bounded modification of the local volatility function:

$$
\left(\min \left\{\max \{x, 0\}, K_{1}\right\}\right)^{\beta} \text {. }
$$

Then, we are easily able to apply our asymptotic expansion to this modified $\lambda$-SABR model up to an arbitrary order. In fact, because we can take $K_{1}$ and $K_{2}$ as arbitrarily large constants, and $K_{3}$ as arbitrarily positive small constant, we may use the same asymptotic expansion both for (47) and (48) as long as the deterministic process $\left\{\left.S^{(\epsilon)}(t)\right|_{\epsilon=0}: 0 \leq t \leq T\right\}$. is in the range between $K_{2}$ and $K_{3}$. If necessary, we could modify the volatility process as well.

The similar modification and consideration could be applied to the asymptotic expansions appearing in the current paper.

\subsubsection{Log-Normal Asymptotic Expansion}

Let us take a log-normal asymptotic expansion for the underlying asset price $S$. That is, for $x_{1}>0$, set $C\left(x_{1}, x_{2}\right)=\left(\log \left(x_{1} / S_{0}\right), x_{2}\right)$ and $\tilde{S}_{t}=\log \frac{S_{t}}{S_{0}}$. Then, we obtain

$$
\begin{aligned}
& d \tilde{S}_{t}=-\frac{1}{2} \sigma_{t}^{2} e^{2(\beta-1) \tilde{S}_{t}} d t+\sigma_{t} e^{(\beta-1) \tilde{S}_{t}} d W_{t}^{1} ; \tilde{S}_{0}=0 \\
& d \sigma_{t}=\lambda\left(\theta-\sigma_{t}\right) d t+\nu \sigma_{t}\left(\rho d W_{t}^{1}+\sqrt{1-\rho^{2}} d W_{t}^{2}\right) ; \sigma_{0}>0
\end{aligned}
$$

Next, we introduce a perturbation $\epsilon \in[0,1]$, again as follows:

$$
\begin{aligned}
& d \tilde{S}_{t}^{(\epsilon)}=-\frac{\eta_{1}(\epsilon)}{2} \sigma^{2} e^{2(\beta-1) \tilde{S}_{t}^{(\epsilon)}} d t+\epsilon \sigma e^{(\beta-1) \tilde{S}_{t}^{(\epsilon)}} d W_{t}^{1} ; \tilde{S}_{0}=0, \\
& d \sigma_{t}^{(\epsilon)}=\eta_{2}(\epsilon) \lambda\left(\theta-\sigma_{t}^{(\epsilon)}\right) d t+\epsilon \nu \sigma_{t}^{(\epsilon)}\left(\rho d W_{t}^{1}+\sqrt{1-\rho^{2}} d W_{t}^{2}\right) ; \sigma_{0}^{(\epsilon)}=\sigma_{0},
\end{aligned}
$$

where $\eta_{i}(\epsilon)=\epsilon^{j_{i}}, i=1,2$ and $j_{i}$ is a nonnegative integer. For instance, typical cases are given below: 


\section{Case I}

$$
\begin{aligned}
& d \tilde{S}_{t}^{(\epsilon)}=-\frac{1}{2} \sigma_{t}^{(\epsilon)^{2}} e^{2(\beta-1) \tilde{S}_{t}^{(\epsilon)}} d t+\epsilon \sigma_{t}^{(\epsilon)} e^{(\beta-1) \tilde{S}_{t}^{(\epsilon)}} d W_{t}^{1} \\
& d \sigma_{t}^{(\epsilon)}=\lambda\left(\theta-\sigma_{t}^{(\epsilon)}\right) d t+\epsilon \nu \sigma_{t}^{(\epsilon)}\left(\rho d W_{t}^{1}+\sqrt{1-\rho^{2}} d W_{t}^{2}\right)
\end{aligned}
$$

Case II (an extension of the the log-normal asymptotic expansion in [49], [42])

$$
\begin{aligned}
& d \tilde{S}_{t}^{(\epsilon)}=-\frac{\epsilon}{2} \sigma_{t}^{(\epsilon)^{2}} e^{2(\beta-1) \tilde{S}_{t}^{(\epsilon)}} d t+\epsilon \sigma_{t}^{(\epsilon)} e^{(\beta-1) \tilde{S}_{t}^{(\epsilon)}} d W_{t}^{1} \\
& d \sigma_{t}^{(\epsilon)}=\lambda\left(\theta-\sigma_{t}^{(\epsilon)}\right) d t+\epsilon \nu \sigma_{t}^{(\epsilon)}\left(\rho d W_{t}^{1}+\sqrt{1-\rho^{2}} d W_{t}^{2}\right)
\end{aligned}
$$

Case III (an extension of [39] to the CEV-type local volatility)

$$
\begin{aligned}
& d \tilde{S}_{t}^{(\epsilon)}=-\frac{\epsilon^{2}}{2} \sigma_{t}^{(\epsilon)^{2}} e^{2(\beta-1) \tilde{S}_{t}^{(\epsilon)}} d t+\epsilon \sigma_{t}^{(\epsilon)} e^{(\beta-1) \tilde{S}_{t}^{(\epsilon)}} d W_{t}^{1} \\
& d \sigma_{t}^{(\epsilon)}=\lambda\left(\theta-\sigma_{t}^{(\epsilon)}\right) d t+\epsilon \nu \sigma_{t}^{(\epsilon)}\left(\rho d W_{t}^{1}+\sqrt{1-\rho^{2}} d W_{t}^{2}\right)
\end{aligned}
$$

An approximation formula of the call price with strike $K$ and maturity $T$ is given as

$$
\begin{aligned}
\operatorname{Call}(K, T) & =\mathbf{E}\left[\left(S_{T}-K\right)_{+}\right]=\mathbf{E}\left[\left(S_{0} \exp \left(G^{(1)}+\tilde{S}_{T}^{(0)}\right)-K\right)_{+}\right] \\
& \approx \int_{y}^{\infty}\left(S_{0} \exp \left(x+\tilde{S}_{T}^{(0)}\right)-K\right) f_{G^{(1)}, N}(x) d x \\
y & =C(K)-\tilde{S}_{T}^{(0)}=\log \frac{K}{S_{0}}-\tilde{S}_{T}^{(0)} .
\end{aligned}
$$

Again, we note that Case I $\left(\eta(\epsilon)=\epsilon^{0}=1\right)$ is harder to be evaluated than the other cases, which results from difficulty in computation of $\tilde{S}_{t}^{(0)}$ for $\eta(\epsilon)=1$.

\subsubsection{CEV Asymptotic Expansion}

Let us take the change of variable function $C$ as $C(x)=\left(C_{1}\left(x_{1}\right), x_{2}\right)$ for $x=\left(x_{1}, x_{2}\right)$, where for $x>0$ and $\beta \in[0,1)$,

$$
C_{1}(x)=\frac{1}{1-\beta} \frac{x^{1-\beta}}{S_{0}^{1-\beta}}\left(=\int^{x} \frac{d z}{z^{\beta} S_{0}^{1-\beta}}\right) .
$$

That is,

$$
C_{1}^{-1}(\tilde{x})=S_{0}(1-\beta)^{\frac{1}{(1-\beta)}} \tilde{x}^{\frac{1}{(1-\beta)}}
$$

Then, as $\tilde{S}_{t}=C_{1}\left(S_{t}\right)$, we have

$$
\begin{aligned}
& d \tilde{S}_{t}=-\frac{1}{2} \frac{\beta}{1-\beta} \sigma_{t}^{2} \frac{1}{\tilde{S}_{t}} d t+\sigma_{t} d W_{t}^{1} ; \tilde{S}_{0}=\frac{1}{1-\beta} \\
& d \sigma_{t}=\lambda\left(\theta-\sigma_{t}\right) d t+\nu \sigma_{t}\left(\rho d W_{t}^{1}+\sqrt{1-\rho^{2}} d W_{t}^{2}\right) \sigma_{0}>0
\end{aligned}
$$

Again, we obtain a perturbed process as follows:

$$
\begin{aligned}
& d \tilde{S}_{t}^{(\epsilon)}=-\frac{\eta_{1}(\epsilon)}{2} \frac{\beta}{1-\beta}\left(\sigma_{t}^{(\epsilon)}\right)^{2} \frac{1}{\tilde{S}_{t}^{(\epsilon)}} d t+\epsilon \sigma_{t}^{(\epsilon)} d W_{t}^{1} ; \tilde{S}_{0}^{(\epsilon)}=\frac{1}{1-\beta} \\
& d \sigma_{t}^{(\epsilon)}=\eta_{2}(\epsilon) \lambda\left(\theta-\sigma_{t}^{(\epsilon)}\right) d t+\epsilon \nu \sigma_{t}^{(\epsilon)}\left(\rho d W_{t}^{1}+\sqrt{1-\rho^{2}} d W_{t}^{2}\right) ; \sigma_{0}^{(\epsilon)}=\sigma_{0},
\end{aligned}
$$


where $\eta_{i}(\epsilon)=\epsilon^{j_{i}}, i=1,2$ and $j_{i}$ is a nonnegative integer.

For illustrative purpose, let us set $\eta_{1}(\epsilon)=\eta_{2}(\epsilon)=\epsilon$. That is,

$$
\begin{aligned}
& d \tilde{S}_{t}^{(\epsilon)}=-\frac{\epsilon}{2} \frac{\beta}{1-\beta}\left(\sigma_{t}^{(\epsilon)}\right)^{2} \frac{1}{\tilde{S}_{t}^{(\epsilon)}} d t+\epsilon \sigma_{t}^{(\epsilon)} d W_{t}^{1} ; \tilde{S}_{0}^{(\epsilon)}=\frac{1}{1-\beta} \\
& d \sigma_{t}^{(\epsilon)}=\epsilon \lambda\left(\theta-\sigma_{t}^{(\epsilon)}\right) d t+\epsilon \nu \sigma_{t}^{(\epsilon)}\left(\rho d W_{t}^{1}+\sqrt{1-\rho^{2}} d W_{t}^{2}\right) ; \sigma_{0}^{(\epsilon)}=\sigma_{0} .
\end{aligned}
$$

In this case, as $\tilde{S}_{t}^{(0)}=\frac{1}{1-\beta}$ and $\sigma_{t}^{(0)}=\sigma_{0}$ for all $t \in[0, T]$, the first term in the asymptotic expansion, $g_{1 t}=\left.\frac{\partial}{\partial \epsilon}\right|_{\epsilon=0} \tilde{S}_{t}^{(\epsilon)}$ follows a Gaussian process:

$$
d g_{1 t}=\frac{-\beta \sigma_{0}^{2}}{2} d t+\sigma_{0} d W_{t}^{1} ; g_{10}=0
$$

Then, by applying Itô's formula to

$$
\hat{g}_{1 t}:=C_{1}^{-1}\left(g_{1 t}\right)=S_{0}(1-\beta)^{\frac{1}{(1-\beta)}} g_{1 t}^{\frac{1}{(1-\beta)}}
$$

and using

$$
g_{1 t}=\frac{1}{1-\beta} \frac{\hat{g}_{1 t}^{1-\beta}}{S_{0}^{1-\beta}}
$$

we formally obtain the dynamics of $\hat{g}_{1 t}$ though it is well-defined only for $g_{1 t} \geq 0$ :

$$
d \hat{g}_{1 t}=\frac{\sigma_{0}^{2}}{2} \hat{g}_{1 t}^{\beta}\left[-\beta S_{0}^{1-\beta}+S_{0}^{2(1-\beta)} \hat{g}_{1 t}^{\beta-1}\right] d t+\sigma_{0} S_{0}^{1-\beta} \hat{g}_{1 t}^{\beta} d W_{t}^{1} ; \hat{g}_{10}=0 .
$$

Here, the diffusion coefficient of $\hat{g}_{1 t}=C_{1}^{-1}\left(g_{1 t}\right)$ is given by $\sigma_{0} S_{0}^{1-\beta}\left(\hat{g}_{1 t}\right)^{\beta}$. As we may think that $S$ is expanded around $\hat{g}_{1}$, we call this case a CEV asymptotic expansion (though $\hat{g}_{1}$ is not exactly a CEV process).

In particular, when $\beta=1 / 2$,

$$
d \hat{g}_{1 t}=\frac{\sigma_{0}^{2}}{2}\left[-\sqrt{S_{0} \hat{g}_{1 t}} / 2+S_{0}\right] d t+\sigma_{0} \sqrt{S_{0} \hat{g}_{1 t}} d W_{t}^{1} ; \hat{g}_{10}=0
$$

and because

$$
\hat{g}_{1 T}=\frac{S_{0}}{4} g_{1 T}^{2},
$$

$\hat{g}_{1 T}$ follows a non-central $\chi$-square distribution, around which the original underlying asset price $S_{T}$ is expanded.

Finally, for $\eta_{i}(\epsilon)=\epsilon^{j_{i}}, i=1,2$ and $j_{i}$ is a nonnegative integer, an approximation formula of the call price with strike $K$ and maturity $T$ is obtained as follows:

$$
\begin{aligned}
\operatorname{Call}(K, T) & =\mathbf{E}\left[\left(S_{T}-K\right)_{+}\right]=\mathbf{E}\left[C_{1}^{-1}\left(\tilde{S}_{T}\right)-K\right] \\
& =\mathbf{E}\left[\left(\left\{S_{0}(1-\beta)^{\frac{1}{(1-\beta)}}\left(\tilde{S}_{T}\right)^{\frac{1}{(1-\beta)}}\right\}-K\right)_{+}\right] \\
& =\mathbf{E}\left[\left(\left\{S_{0}(1-\beta)^{\frac{1}{(1-\beta)}}\left(\tilde{S}_{T}^{(1)}\right)^{\frac{1}{(1-\beta)}}\right\}-K\right)_{+}\right] \\
& =\mathbf{E}\left[\left(\left\{S_{0}(1-\beta)^{\frac{1}{(1-\beta)}}\left(G^{(1)}+\tilde{S}_{T}^{(0)}\right)^{\frac{1}{(1-\beta)}}\right\}-K\right)_{+}\right] \\
& \approx \int_{y}^{\infty}\left(\left\{S_{0}(1-\beta)^{\frac{1}{(1-\beta)}}\left(x+\tilde{S}_{T}^{(0)}\right)^{\frac{1}{(1-\beta)}}\right\}-K\right) f_{G^{(1)}, N}(x) d x \\
y & =C_{1}(K)-\tilde{S}_{T}^{(0)}=\frac{1}{1-\beta}\left(\frac{K}{S_{0}}\right)^{1-\beta}-\tilde{S}_{T}^{(0)} .
\end{aligned}
$$




\subsection{Numerical Examination}

For numerical examination of approximation for European option prices, we take SABR [10] model ( $\lambda$ SABR with $\lambda=0$ ):

$$
\begin{aligned}
& d S_{t}=\sigma_{t}\left(S_{t}^{\beta} S_{0}^{1-\beta}\right) d W_{t}^{1} ; S_{0}>0, \\
& d \sigma_{t}=\nu \sigma_{t}\left(\rho d W_{t}^{1}+\sqrt{1-\rho^{2}} d W_{t}^{2}\right) ; \sigma_{0}>0
\end{aligned}
$$

In particular, we apply the following three different expansions for approximation. (Although we use the same notation $f_{G^{(1)}, N}(x)$ for the density approximations in all expansions, each represents the density obtained by the corresponding expansion.)

1. Normal expansion

This case corresponds to the original asymptotic expansion method.(e.g. [34]). We apply the asymptotic expansion to the following perturbed stochastic differential equation:

$$
\begin{aligned}
& d S_{t}^{(\epsilon)}=\epsilon \sigma_{t}\left(S_{t}^{(\epsilon)}\right)^{\beta} S_{0}^{1-\beta} d W_{t}^{1} ; S_{0}^{(\epsilon)}=S_{0}>0 \\
& d \sigma_{t}^{(\epsilon)}=\epsilon \nu \sigma_{t}^{(\epsilon)}\left(\rho d W_{t}^{1}+\sqrt{1-\rho^{2}} d W_{t}^{2}\right) ; \sigma_{0}^{(\epsilon)}=\sigma_{0}>0
\end{aligned}
$$

Then, an approximation of a call option price with maturity $T$ and strike price $K$ is given by

$$
\begin{aligned}
C(K, T) & \approx \int_{y}^{\infty}(x-y) f_{G^{(1)}, N}(x) d x \\
y & =K-S_{T}^{(0)}=K-S_{0}
\end{aligned}
$$

where $G^{(1)}=\left.G^{(\epsilon)}\right|_{\epsilon=1}$,

$$
G^{(\epsilon)}=\frac{S_{T}^{(\epsilon)}-S_{T}^{(0)}}{\epsilon}=\frac{S_{T}^{(\epsilon)}-S_{0}}{\epsilon},
$$

and $f_{G^{(1)}, N}$ denotes the asymptotic expansion of density of $G^{(\epsilon)}$ up to $\epsilon^{N}$-th order evaluated at $\epsilon=1$. Integrals may be calculated by the formulas:

$$
\int_{y}^{\infty}(x-y) H_{k}(x ; \Sigma) f_{g_{1 T}}(x) d x=\Sigma^{2} H_{k-2}(-y ; \Sigma) f_{g_{1 T}}(y) .
$$

2. Log-normal expansion

We apply the expansion result in Section 3.2.1 with $\eta_{1}(\epsilon)=\epsilon$ :

$$
\begin{aligned}
& d \tilde{S}_{t}^{(\epsilon)}=-\frac{\epsilon}{2} \sigma_{t}^{(\epsilon)^{2}} e^{2(\beta-1) \tilde{S}_{t}^{(\epsilon)}} d t+\epsilon \sigma_{t}^{(\epsilon)} e^{(\beta-1) \tilde{S}_{t}^{(\epsilon)}} d W_{t}^{1} ; \tilde{S}_{0}^{(\epsilon)}=0, \\
& d \sigma_{t}^{(\epsilon)}=\epsilon \nu \sigma_{t}^{(\epsilon)}\left(\rho d W_{t}^{1}+\sqrt{1-\rho^{2}} d W_{t}^{2}\right) ; \sigma_{0}^{(\epsilon)}=\sigma_{0}
\end{aligned}
$$

In this case, an approximation of a call option price with maturity $T$ and strike price $K$ is given by

$$
\begin{aligned}
\operatorname{Call}(K, T) & \approx \int_{y}^{\infty}\left(S_{0} e^{x}-K\right) f_{G^{(1)}, N}(x) d x \\
y & =C(K)-\tilde{S}_{T}^{(0)}=\log \frac{K}{S_{0}} .
\end{aligned}
$$

3. CEV expansion

We apply the result in Section 3.2.2 with $\eta_{1}(\epsilon)=\epsilon$, that is,

$$
\begin{aligned}
& d \tilde{S}_{t}^{(\epsilon)}=-\frac{\epsilon}{2} \frac{\beta}{1-\beta}\left(\sigma_{t}^{(\epsilon)}\right)^{2} \frac{1}{\tilde{S}_{t}^{(\epsilon)}} d t+\epsilon \sigma_{t}^{(\epsilon)} d W_{t}^{1} ; \tilde{S}_{0}^{(\epsilon)}=\frac{1}{1-\beta}, \\
& d \sigma_{t}^{(\epsilon)}=\epsilon \nu \sigma_{t}^{(\epsilon)}\left(\rho d W_{t}^{1}+\sqrt{1-\rho^{2}} d W_{t}^{2}\right) ; \sigma_{0}^{(\epsilon)}=\sigma_{0} .
\end{aligned}
$$


Hence, an approximation formula of the call price with strike $K$ and maturity $T$ is obtained as follows:

$$
\begin{aligned}
\operatorname{Call}(K, T) & \approx \int_{y}^{\infty}\left(\left\{S_{0}(1-\beta)^{\frac{1}{(1-\beta)}}\left(\frac{1}{(1-\beta)}+x\right)^{\frac{1}{(1-\beta)}}\right\}-K\right) f_{G^{(1)}, N}(x) d x \\
y & =C_{1}(K)-\tilde{S}_{T}^{(0)}=\frac{1}{1-\beta}\left(\frac{K}{S_{0}}\right)^{1-\beta}-\frac{1}{(1-\beta)}
\end{aligned}
$$

In the numerical examples below, we set the parameters as follows:

- The option maturity $T$, the current underlying asset price $S_{0}$, the current volatility $\sigma_{0}$, the volatility on volatility $\nu$ :

$$
S_{0}=100, T=1, \sigma_{0}=0.30, \nu=0.30
$$

- The instantaneous correlation $\rho$ between the asset price $S$ and its volatility $\sigma$ : three different correlations;

$$
\rho=0.0,-0.5,-0.75 \text {. }
$$

- The CEV parameter $\beta$ of the underlying asset price process $S$ : nine different $\beta \mathrm{s}$;

$$
\beta=0.0,0.125,0.25,0.375,0.50,0.625,0.75,0.875,1.0 .
$$

- Strike price $K$ of the option: twenty different strikes;

$$
K=10,20, \cdots, 100,110,120, \cdots, 200 .
$$

Benchmark prices are computed by Monte Carlo simulation with $10^{8}$ trials, 1024 time steps and the antithetic variable method, where Euler-Maruyama scheme is used for the discretization of the stochastic differential equation (73). Then, the absolute approximation error is given by |(approximation price) (benchmark price)| for each case. We compute each expansion up to the third order. That is, for each approximation we use $\epsilon^{j}$-order expansion, $f_{G^{(1)}, j}(x)(j=1,2,3)$ for the density $f_{G^{(1)}}(x)$ :

$$
f_{G^{(1)}, j}(x)=f_{g_{1 T}}(x)+\sum_{n=1}^{j}\left(\sum_{m=0}^{3 n} C_{n m} H_{m}\left(x-C, \Sigma_{T}\right)\right) f_{g_{1 T}}(x) .
$$

For each expansion, the higher order expansion provides the better approximation. Particularly, as for the third order ( $\epsilon^{3}$-order) expansion Figure 1-3 below show the average values of the absolute approximation errors for option prices with all the strikes in (87) for each $\beta$ in (86), given the correlation value $\rho$ in (85). In the figures, the horizontal axis is $\beta$ while the vertical axis is the average absolute error; Normal A.E. 3rd, Log A.E. 3rd and LV A.E. 3rd represent normal expansion, log-normal expansion and CEV expansion, respectively. Because CEV expansion is not well-defined for $\beta=1$, we use the same formula as the one of log-normal expansion.

We find that $C E V$ expansion provides the most stable approximations for all the cases. On the other hand, log-normal expansion is not robust to the change in $\beta$ in a sense that its approximation becomes worse as $\beta$ deviates from 1. As for normal expansion, although its approximation in zero correlation $\rho=0.0$ (Figure 1) becomes worse as $\beta$ deviates from 0 , it becomes stable for the higher (negative) correlations such as $\rho=-0.5,-0.75$ (in Figure 2, 3, respectively). For completeness, the appendix provides the results of the first ( $\epsilon$-order) and second order $\left(\epsilon^{2}\right.$-order) expansions. Through investigation of the behavior of the the asymptotic expansions up to the third order, we observe that $C E V$ expansion becomes more precise with the same level of absolute errors across the whole range of $\beta$ along the higher order expansions. Thus, we expect a higher order $C E V$ expansion will produce the better and more stable approximation than normal and log-normal expansions. 


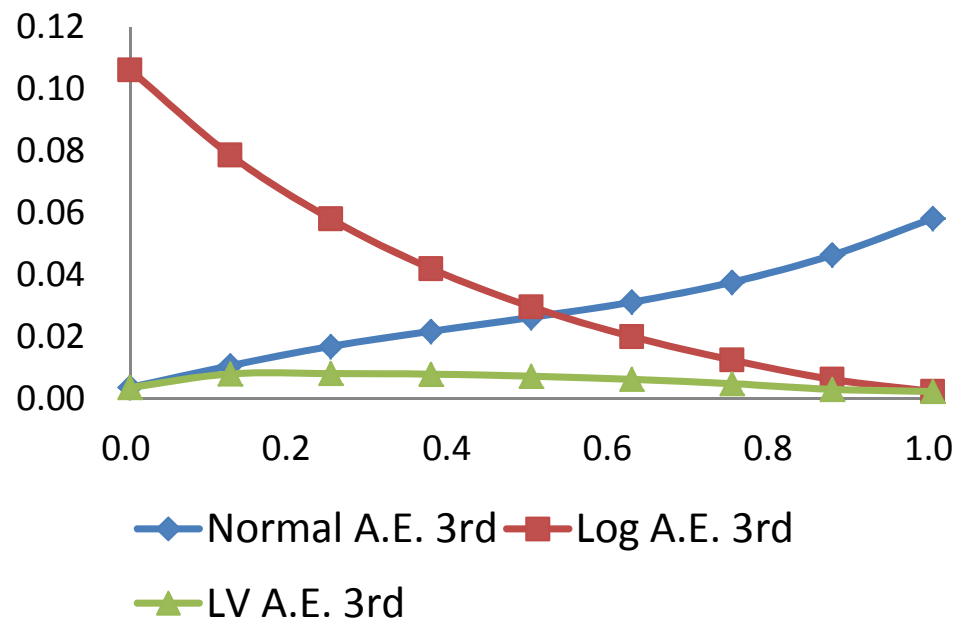

Figure 1: Average value of the absolute errors of the third order expansion for option prices with twenty different strikes in (87) for the case of correlation $\rho=0.0$. The $\mathrm{x}$-axis indicates $\beta$ and the $\mathrm{y}$-axis the average of the absolute approximation errors. Normal A.E. 3rd, Log A.E. 3rd and LV A.E. 3rd represent the third order $\left(\epsilon^{3}\right.$-order $)$ normal expansion, log-normal expansion and $C E V$ expansion, respectively. Because $C E V$ expansion is not well-defined for $\beta=1$, we use the same formula as the one of log-normal expansion. Note that $C E V$ expansion provides the most stable approximations, while normal expansion and log-normal expansion do not give robust approximations against the change in $\beta$.

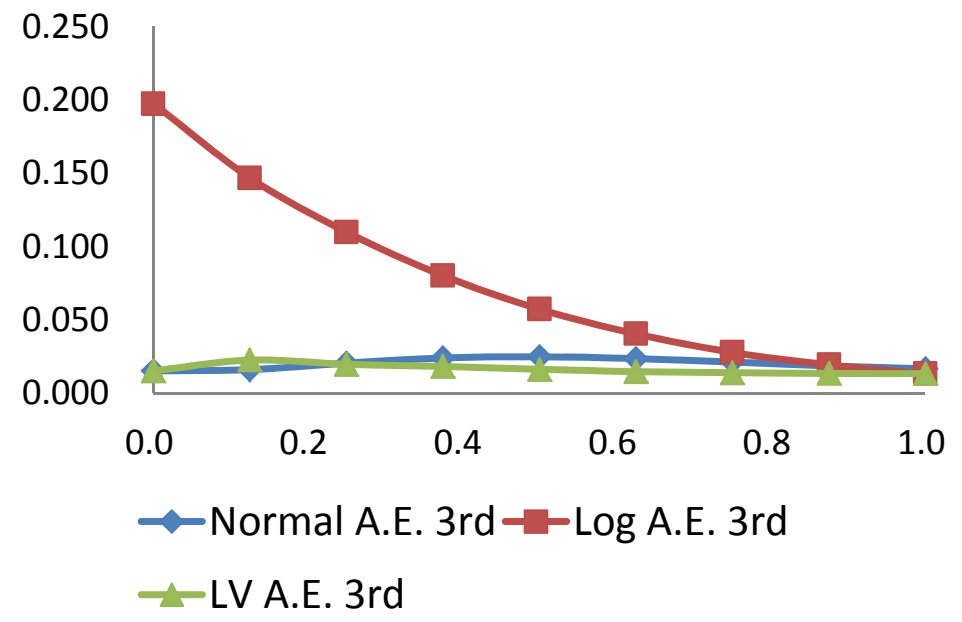

Figure 2: Average value of the absolute errors of the third order expansion for option prices with twenty different strikes in (87) for the case of correlation $\rho=-0.5$. Again, $C E V$ expansion generates the stable approximations, and log-normal expansion does not give robust approximations against the change in $\beta$. On the other hand, normal expansion provides more stable approximations than in the case of correlation $\rho=0.0$. 


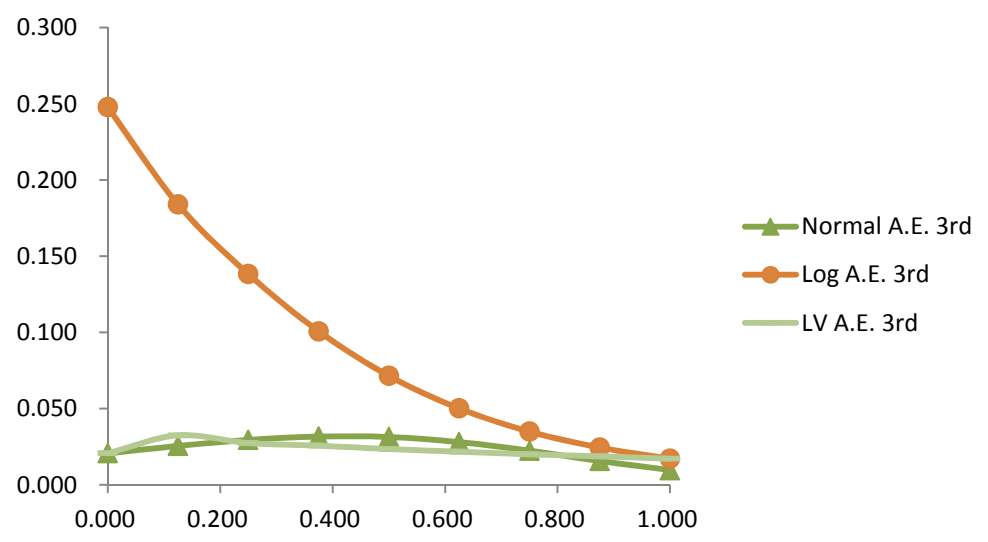

Figure 3: Average value of the absolute errors of the third order expansion for option prices with twenty different strikes in (87) for the case of correlation $\rho=-0.75$. The same observation holds as in the case of correlation $\rho=-0.5$.

\section{Conclusion}

This paper has constructed a new scheme that enables us to set a distribution around which we would like to expand a target random variable, and to approximate the target variable up to any order based upon the distribution. Particularly, the present method has extended a general computational scheme proposed by our previous results [49], [42], and [43]. As numerical examples, we have shown new Log-normal and $C E V$ expansions up to the third order for approximations of option prices under SABR model, which demonstrates that the $C E V$ expansion will be a candidate for a more precise and robust technique than other approximation schemes.

\section{References}

[1] Bayer,C., Laurence, P. [2012], "Asymptotics beats Monte Carlo: The case of correlated local vol baskets," forthcoming in Communications on Pure and Applied Mathematics.

[2] Ben Arous, G., Laurence, P.[2009],“ Second order expansion for implied volatility in two factor local stochastic volatility models and applications to the dynamic $\lambda$-SABR model," preprint.

[3] Bichteler, K., Gravereaux, J.-B. and Jacod, J. [1987], Malliavin Calculus for Processes with Jumps, volume 2 of Stochastic Monographs, Gordon and Breach Science Publishers.

[4] Cox, J. [1975], "Notes on Option Pricing I: Constant Elasticity of Diffusions." Unpublished draft, Stanford University.

[5] Fujii, M,, Takahashi, A.[2012], "Analytical Approximation for Non-linear FBSDEs with Perturbation Scheme," International Journal of Theoretical and Applied Finance, Vol. 15, No. 5, DOI: 10.1142/S0219024912500343.

[6] Fujii, M,, Takahashi, A. [2012], Perturbative Expansion Technique for Non-linear FBSDEs with Interacting Particle Method," Working paper, CARF-F-278, the University of Tokyo.

[7] Fujii, M,, Takahashi, A.[2012], "Perturbative Expansion of FBSDE in an Incomplete Market with Stochastic Volatility," Quarterly Journal of Finance Vol.2, No.3, 1250015, DOI:10.1142/S2010139212500152. 
[8] Fouque, J. P., Papanicolaou,G., Sircar, K.R. [2000], Derivatives in Financial Markets with Stochastic Volatility, Cambridge University Press.

[9] Gatheral, J., Hsu, E.P., Laurence, P., Ouyang, C., Wang, T-H.[2009], "Asymptotics of implied volatility in local volatility models," Mathematical Finance, 22(4), 2012, 591-620.

[10] Hagan, P.S., Kumar, D., Lesniewskie, A.S.,Woodward, D.E. [2002], Managing Smile Risk, Willmott Magazine, 2002, 84-108.

[11] Ikeda, N., Watanabe, S. [1989], Stochastic Differential Equations and Diffusion Processes, Second Edition, North-Holland/Kodansha, Tokyo.

[12] Kato, T., Takahashi, A., Yamada. T.[2012], An Asymptotic Expansion for Solutions of CauchyDirichlet Problem for Second Order ParabolicPDEs and its Application to Pricing Barrier Options, Working paper, CARF-F-271, the University of Tokyo.

[13] Kunitomo, N., Takahashi, A.[1992], Pricing Average Options, Japan Financial Review, Vol.14, 1992, 1-20(in Japanese).

[14] Kunitomo, N., Takahashi, A. [2001], "The Asymptotic Expansion Approach to the Valuation of Interest Rate Contingent Claims," Mathematical Finance, Vol. 11, 117-151.

[15] Kunitomo, N., Takahashi, A., [2003], On Validity of the Asymptotic Expansion Approach in Contingent Claim Analysis, Annals of Applied Probability, Vol.13(3), 914-95.

[16] Kunitomo, N., Takahashi, A.[2004], "Applications of the Asymptotic Expansion Approach based on Malliavin-Watanabe Calculus in Financial Problems," Stochastic Processes and Applications to Mathematical Finance: Proceedings of the Ritsumeikan International Symposium 2003, (Akahori, J., Ogawa, S., Watanabe, S. ed.), 195-232, World Scientific.

[17] Kusuoka S., Osajima, Y. [2008], "A Remark on the Asymptotic Expansion of Density Function of Wiener Functionals," Journal of Functional Analysis, Vol.255-9, 2545-2562.

[18] Kusuoka S, Strook, D. [1991], "Precise Asymptotics of Certain Wiener Functionals," Journal of Functional Analysis, Vol.99, 1-74.

[19] Henry-Labordère, P.[2008], Analysis, Geometry and Modeling in Finance : Advanced Methods in Options Pricing, Chapman and Hall.

[20] Li, C. [2010], "Closed-form Expansion, Conditional Expectation, and Option Valuation," SSRN Working Paper Series.

[21] Malliavin, P. [1997], Stochastic Analysis, Springer.

[22] Malliavin, P, Thalmaier, A. [2006], Stochastic Calculus of Variations in Mathematical Finance, Springer.

[23] Matsuoka, R. Takahashi, A., Uchida, Y. [2004], "A New Computational Scheme for Computing Greeks by the Asymptotic Expansion Approach," Asia-Pacific Financial Markets, Vol.11, 393-430.

[24] Nualart, D. [1995], The Malliavin Calculus and Related Topics, Springer.

[25] Nualart, D., Üstünel A. S. and Zalai M. [1988], "On the moments of a multiple Wiener-Itô integral and the space induced by the polynomials of the integral," Stochastics, Vol 25, 233-340.

[26] Osajima, Y. [2006], "The Asymptotic Expansion Formula of Implied Volatility for Dynamic SABR Model and FX Hybrid Model," Preprint, Graduate School of Mathematical Sciences, the University of Tokyo.

[27] Osajima, Y. [2007], "General Asymptotics of Wiener Functionals and Application to Mathematical Finance," Preprint, Graduate School of Mathematical Sciences, the University of Tokyo. 
[28] Shiraya, K,, Takahashi, A.[2011], "Pricing Average Options on Commodities," Journal of Futures Markets, Vol.31-5, 407-439.

[29] Shiraya, K,, Takahashi, A. [2012], "Pricing Multi-Asset Cross Currency Options," Journal of Futures Markets, DOI: 10.1002/fut.21590.

[30] Shiraya, K., Takahashi, A., Toda, M. [2012], "Pricing Barrier and Average Options under Stochastic Volatility Environment," Journal of Computational Finance, vol.15-2, winter 2011/12, 111-148.

[31] Shiraya, K,, Takahashi, A., Yamazaki, A. [2011], "Pricing Swaptions under the LIBOR Market Model of Interest Rates with Local-Stochastic Volatility Models," Wilmott Magazine, Volume 2011, Issue 54, pp 61-73.

[32] Shiraya, K,, Takahashi, A., Yamada, T.[2012], "Pricing Discrete Barrier Options under Stochastic Volatility," Asia-Pacific Financial Markets, Volume 19-3 , 205-23.

[33] Siopacha, M., Teichmann, J., "Weak and Strong Taylor Methods for Numerical Solutions of Stochastic Differential Equations," Quantitative Finance, Volume 11-4, 517-528, 2011.

[34] Takahashi, A.[1995], "Essays on the Valuation Problems of Contingent Claims," Ph.D. Dissertation, Haas School of Business, University of California, Berkeley.

[35] Takahashi, A.[[1999], An Asymptotic Expansion Approach to Pricing Contingent Claims, Asia-Pacific Financial Markets, Vol. 6, 115-151.

[36] Takahashi, A. [2009], "On an Asymptotic Expansion Approach to Numerical Problems in Finance," Selected Papers on Probability and Statistics, pp. 199-217, American Mathematical Society.

[37] Takahashi, A., Matsushima, S. [2004], "Monte Carlo Simulation with an Asymptotic Expansion in HJM Framework," FSA Research Review 2004, 82-103, Financial Services Agency (in Japanese).

[38] Takahashi, A, Saito, T. [2003], "An Asymptotic Expansion Approach to Pricing American Options," Monetary and Economic Studies, Vol. 22, 35-87. (in Japanese).

[39] Takahashi, A., Takehara, K.[2007], "Pricing Currency Options with a Market Model of Interest Rates under Jump-Diffusion Stochastic Volatility Processes of Spot Exchange Rates," Asia-Pacific Financial Markets, Vol.14 , pp. 69-121.

[40] Takahashi, A., Takehara, K.[2008], "Fourier Transform Method with an Asymptotic Expansion Approach: an Application to Currency Options," International Journal of Theoretical and Applied Finance, Vol.11-4, 381-401.

[41] Takahashi, A., Takehara, K. [2010], "A Hybrid Asymptotic Expansion Scheme: an Application to Long-term Currency Options,", International Journal of Theoretical and Applied Finance, Vol.13-8, 1179-1221.

[42] Takahashi, A., Takehara, K., Toda. M.[2009], Computation in an Asymptotic Expansion Method, Working paper, CARF-F-149, the University of Tokyo.

[43] Takahashi, A., Takehara, K., Toda. M., "A General Computation Scheme for a High-Order Asymptotic Expansion Method," International Journal of Theoretical and Applied Finance, 15(6), DOI: 10.1142/S0219024912500446, 2012.

[44] Takahashi, A., Uchida, Y. [2006], "New Acceleration Schemes with the Asymptotic Expansion in Monte Carlo Simulation," Advances in Mathematical Economics, Vol. 8, 411-431.

[45] Takahashi, A., Yamada, T.[2012], "An Asymptotic Expansion with Push-Down of Malliavin Weights," SIAM Journal on Financial Mathematics, Volume 3, pp 95-136.

[46] Takahashi, A., Yamada, T.[2012], "A Remark on Approximation of the Solutions to Partial Differential Equations in Finance," Recent Advances in Financial Engineering 2011, pp.133-181. 
[47] Takahashi, A., Yoshida, N. [2004], "An Asymptotic Expansion Scheme for Optimal Investment Problems," Statistical Inference for Stochastic Processes, 7, No.2, 153-188.

[48] Takahashi, A., Yoshida, N. [2005], "Monte Carlo Simulation with Asymptotic Method," The Journal of Japan Statistical Society, Vol. 35-2, 171-203.

[49] Toda, M. [2009], "Notes on an Asymptotic Expansion- Applications and Extensions -," Master Thesis, Graduate School Economics, the University of Tokyo.

[50] Watanabe, S.[1987], "Analysis of Wiener Functionals (Malliavin Calculus) and Its Applications to Heat Kernels", The Annals of Probability, Vol.15, 1-39.

[51] Yamamoto, K. Sato, S., Takahashi, A. [2010], "Probability Distribution and Option Pricing for Drawdown in a Stochastic Volatility Environment," International Journal of Theoretical and Applied Finance, vol.13-2, 335-354.

[52] Yamamoto, K., Takahashi, A. [2009], "A Remark on a Singular Perturbation Method for Option Pricing under a Stochastic Volatility Model," Asia-Pacific Financial Markets, Vol.16-4, 333-345.

[53] Yoshida, N.[1992], "Asymptotic Expansion for Small Diffusions via the Theory of MalliavinWatanabe", Probability Theory and Related Fields, Vol. 92, 275-311.

[54] Yoshida, N.[1992], "Asymptotic Expansions for Statistics Related to Small Diffusions", Journal of Japan Statistical Society, Vol.22, 139-159.

\section{Appendix}

This appendix presents the approximation result of the first ( $\epsilon$-order) and second order ( $\epsilon^{2}$-order) expansions for numerical examples in Section 3.2. CEV expansion provides the best approximation for zero correlation, $\rho=0$, while Normal expansion gives the best for the other correlation cases, $\rho=-0.5,-0.75$. The approximation of log-normal expansion is the worst for all cases.

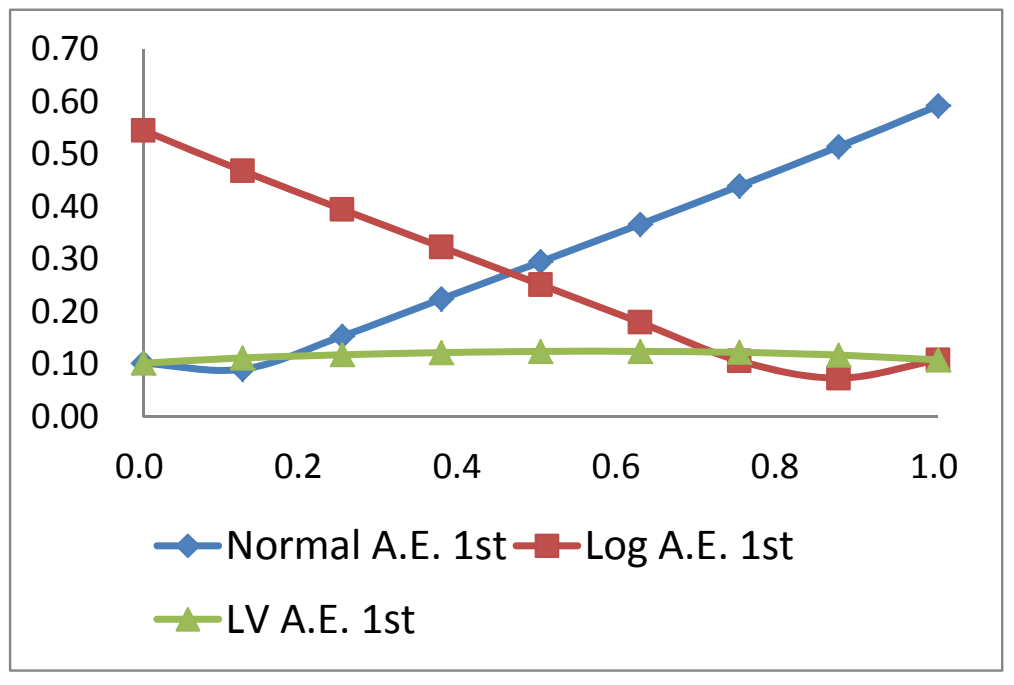

Figure 4: Average value of the absolute errors of the first order expansion for option prices with twenty different strikes in (87) for the case of correlation $\rho=0.0$. The $\mathrm{x}$-axis indicates $\beta$ and the $\mathrm{y}$-axis the average absolute approximation error. Normal A.E. 1st, Log A.E. 1st and LV A.E. 1st represent the first order ( $\epsilon$-order) normal expansion, log-normal expansion and $C E V$ expansion, respectively. 


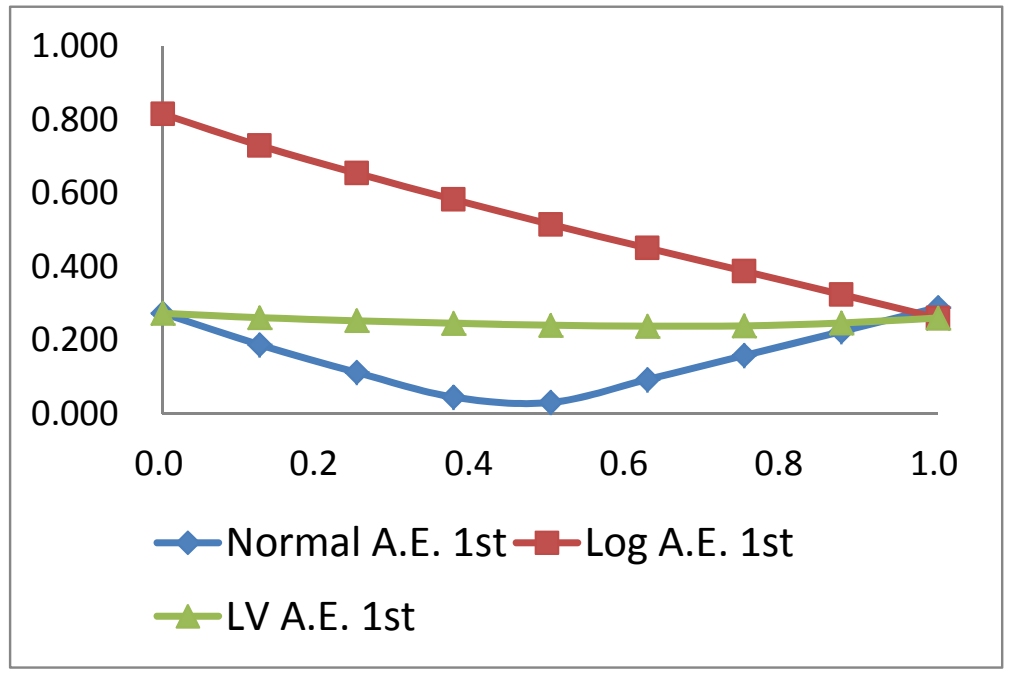

Figure 5: Average value of the absolute errors of the first order expansion for option prices with twenty different strikes in (87) for the case of correlation $\rho=-0.5$.

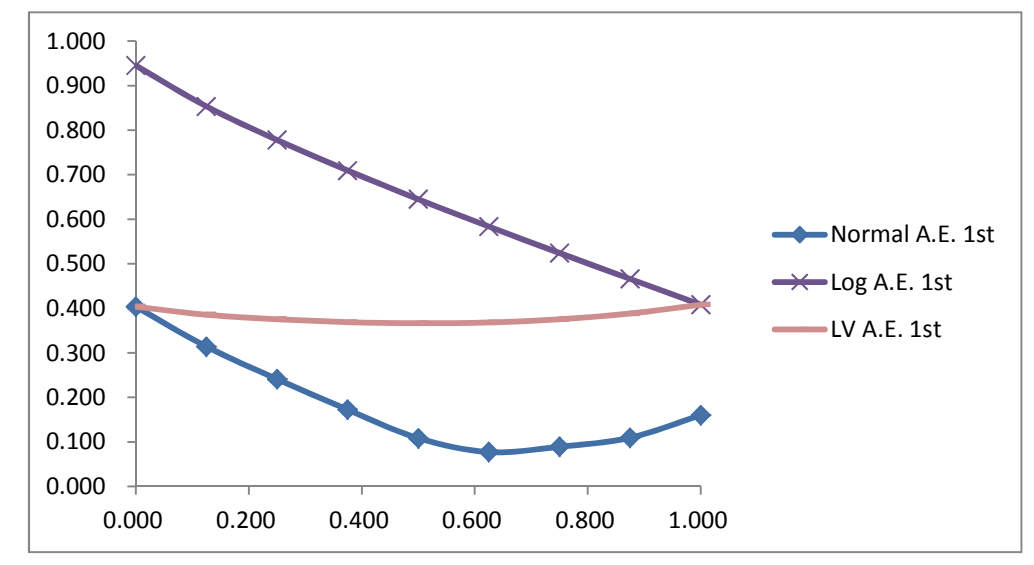

Figure 6: Average value of the absolute errors of the first order expansion for option prices with twenty different strikes for the case of correlation $\rho=-0.75$. 


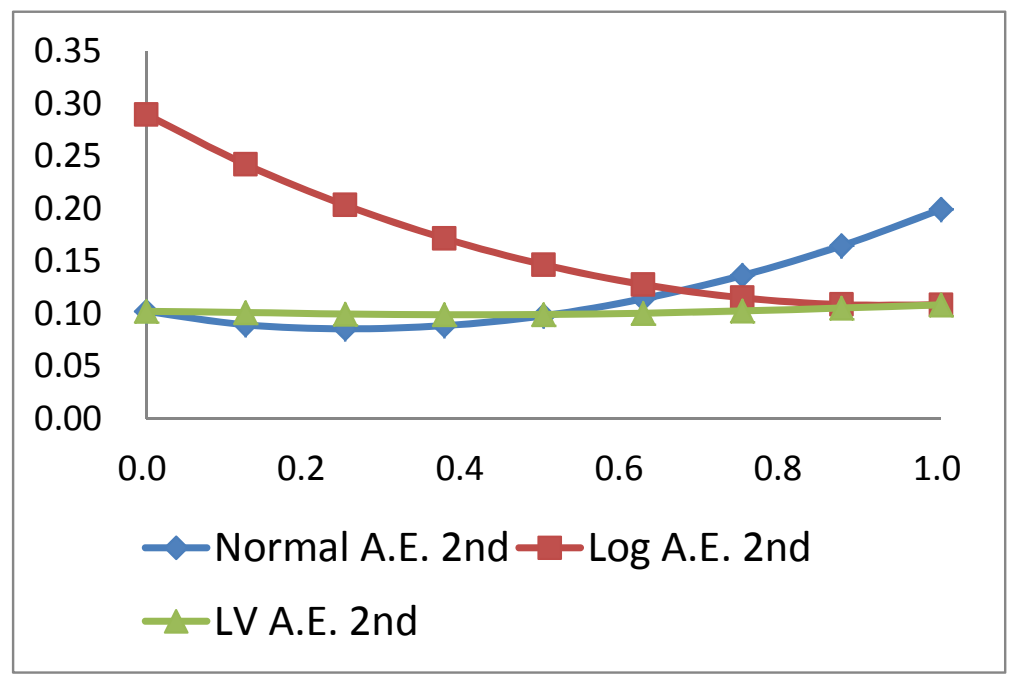

Figure 7: Average value of the absolute errors of the second order expansion for option prices with twenty different strikes in (87) for the case of correlation $\rho=0.0$. The $\mathrm{x}$-axis indicates $\beta$ and the $\mathrm{y}$-axis the average of the absolute approximation errors. Normal A.E. 2nd, Log A.E. 2nd and LV A.E. 2nd represent the second order $\left(\epsilon^{2}\right.$-order) normal expansion, log-normal expansion and CEV expansion, respectively.

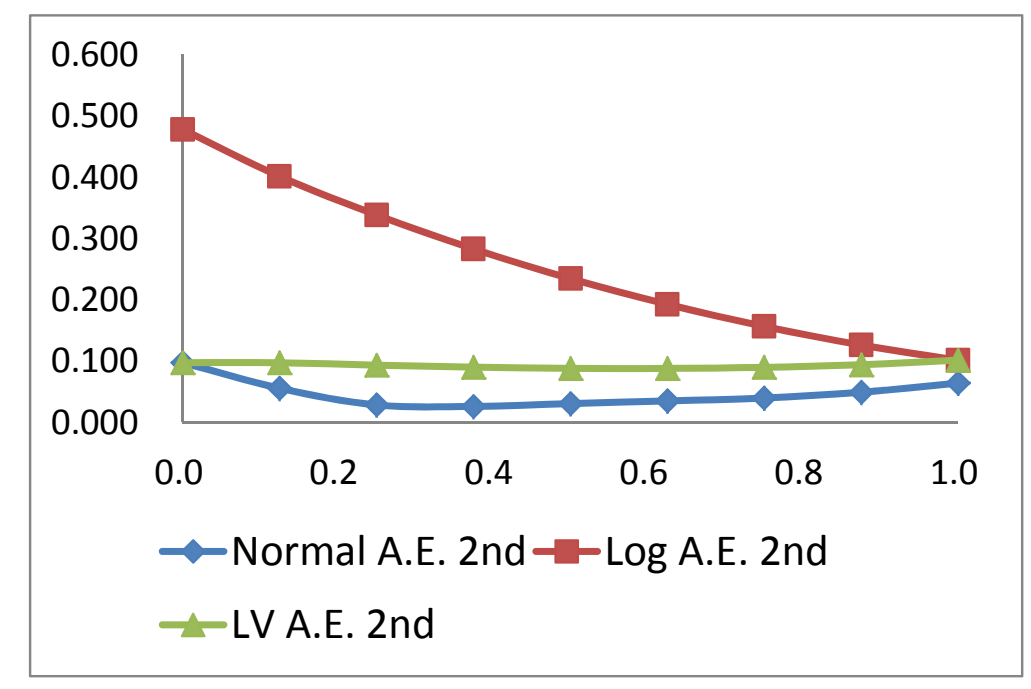

Figure 8: Average value of the absolute errors of the second order expansion for option prices with twenty different strikes in (87) for the case of correlation $\rho=-0.5$. 


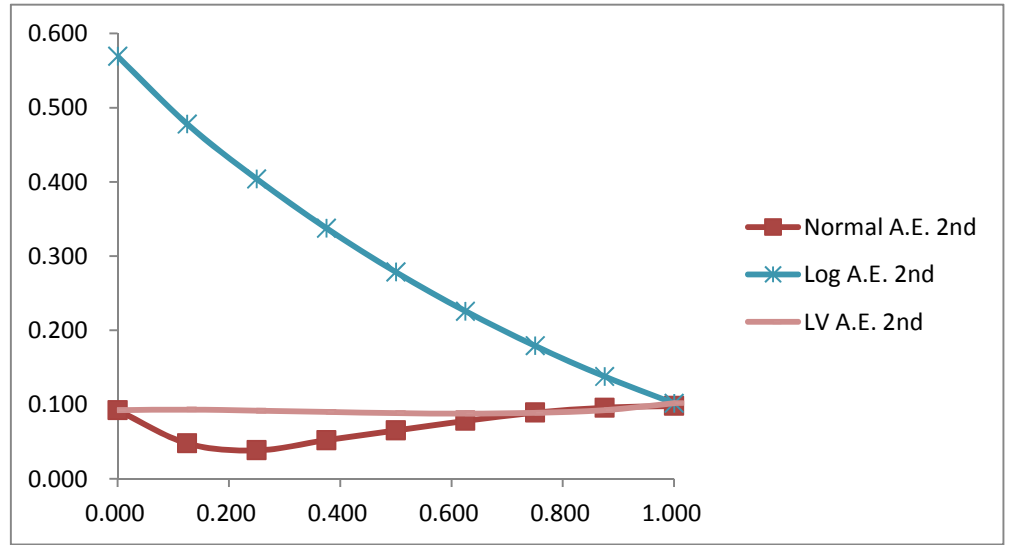

Figure 9: Average value of the absolute errors of the second order expansion for option prices with twenty different strikes in (87) for the case of correlation $\rho=-0.75$. 